\title{
Divergent effects of glucose and fructose on hepatic lipogenesis and insulin signaling
}

\author{
Samir Softic,, ${ }^{1,2}$ Manoj K. Gupta, ${ }^{3}$ Guo-Xiao Wang, ${ }^{1}$ Shiho Fujisaka, ${ }^{1,4}$ Brian T. O'Neill, ${ }^{1,5}$ Tata Nageswara Rao, ${ }^{1,6}$ \\ Jennifer Willoughby, ${ }^{7}$ Carole Harbison, ${ }^{7}$ Kevin Fitzgerald, ${ }^{7}$ Olga Illkayeva, ${ }^{8}$ Christopher B. Newgard, ${ }^{8}$ David E. Cohen, ${ }^{9}$ \\ and C. Ronald Kahn'
}

Joslin Diabetes Center, Harvard Medical School, Boston, Massachusetts, USA. ${ }^{2}$ Boston Children's Hospital, Division of Gastroenterology, Boston, Massachusetts, USA. ${ }^{3}$ Section of Islet Cell and Regenerative Medicine, Joslin Diabetes Center, Boston, Massachusetts, USA. ${ }^{4}$ First Department of Internal Medicine, University of Toyama, Toyama, Japan. ${ }^{5}$ Division of Endocrinology and Metabolism, Fraternal Order of Eagles Diabetes Research Center, University of lowa Carver College of Medicine, lowa City, lowa, USA. ${ }^{E}$ Experimental Hematology, Department of Biomedicine, University Hospital Basel and University of Basel, Basel, Switzerland. '7 Alnylam Pharmaceuticals, Cambridge, Massachusetts, USA. ${ }^{8}$ Sarah W. Stedman Nutrition and Metabolism Center, Duke Molecular Physiology Institute and Departments of Pharmacology and Cancer Biology and Medicine, Duke University Medical Center, Durham, North Carolina, USA. ${ }^{~}$ Division of Gastroenterology and Hepatology, Weill Cornell Medical College, New York, New York, USA.

Overconsumption of high-fat diet (HFD) and sugar-sweetened beverages are risk factors for developing obesity, insulin resistance, and fatty liver disease. Here we have dissected mechanisms underlying this association using mice fed either chow or HFD with or without fructose- or glucose-supplemented water. In chow-fed mice, there was no major physiological difference between fructose and glucose supplementation. On the other hand, mice on HFD supplemented with fructose developed more pronounced obesity, glucose intolerance, and hepatomegaly as compared to glucose-supplemented HFD mice, despite similar caloric intake. Fructose and glucose supplementation also had distinct effects on expression of the lipogenic transcription factors ChREBP and SREBP1c. While both sugars increased ChREBP- $\beta$, fructose supplementation uniquely increased SREBP1c and downstream fatty acid synthesis genes, resulting in reduced liver insulin signaling. In contrast, glucose enhanced total ChREBP expression and triglyceride synthesis but was associated with improved hepatic insulin signaling. Metabolomic and RNA sequence analysis confirmed dichotomous effects of fructose and glucose supplementation on liver metabolism in spite of inducing similar hepatic lipid accumulation. Ketohexokinase, the first enzyme of fructose metabolism, was increased in fructose-fed mice and in obese humans with steatohepatitis. Knockdown of ketohexokinase in liver improved hepatic steatosis and glucose tolerance in fructose-supplemented mice. Thus, fructose is a component of dietary sugar that is distinctively associated with poor metabolic outcomes, whereas increased glucose intake may be protective.

\section{Introduction}

There is a worldwide epidemic of obesity, type 2 diabetes, and metabolic syndrome $(1,2)$. Nonalcoholic fatty liver disease (NAFLD) is a liver manifestation of metabolic syndrome and is estimated to affect one billion individuals worldwide (3). The major risk factor for development of NAFLD is excessive caloric intake, which in Western societies is mainly derived from overconsumption of high-fat foods and increased intake of sugar-sweetened beverages. Indeed, consumption of sugar-sweetened beverages has emerged as a significant risk factor for development of obesity (4), NAFLD (5), and type 2 diabetes (6).

Most foods and beverages are sweetened with table sugar (sucrose) or high-fructose corn syrup, both of which are composed of monosaccharides and disaccharides of glucose and fructose. Despite the fact that fructose and glucose are both 6-carbon sugars, their metabolism is starkly different. Fructose is mainly

Conflict of interest: C.R. Kahn and S. Softic received grant support from and J. Willoughby, C. Harbison, and K. Fitzgerald are employees of Alnylam Pharmaceuticals.

Submitted: April 13, 2017; Accepted: August 10, 2017.

Reference information: / Clin Invest. 2017;127(11):4059-4074.

https://doi.org/10.1172/JCI94585. metabolized by the liver, while glucose is used by every cell in the body. Unlike glucose, which causes robust insulin secretion, fructose alone is not an insulin secretagogue, and its metabolism is not regulated by insulin. Fructose metabolism is more rapid than glucose metabolism (7), which may help to explain why fructose phosphorylation to fructose- 1 phosphate in the liver has been associated with ATP depletion (8). The biggest difference may be in their lipogenic potential, as fructose robustly increases the expression of enzymes involved in fatty acid synthesis (9). Indeed, tracer studies show that fructose is incorporated into both glycerol and free fatty acids, while glucose is not converted into free fatty acids, at least acutely (10).

A primary abnormality in NAFLD may be increased hepatic de novo lipogenesis (9). While both monosaccharides may support lipogenesis, it is unclear whether both sugars lead to the formation of the same types of lipids or whether the magnitude of lipogenesis is the primary difference between fructose versus glucose supplementation. It has been proposed that fructose stimulates lipogenesis via induction of the SREBP1c transcription factor, either directly via upregulation of ER stress (11) or indirectly by inducing insulin resistance and hyperinsulinemia, as insulin activates transcription and proteolytic activation of SREBP1c (12). On the other 
A

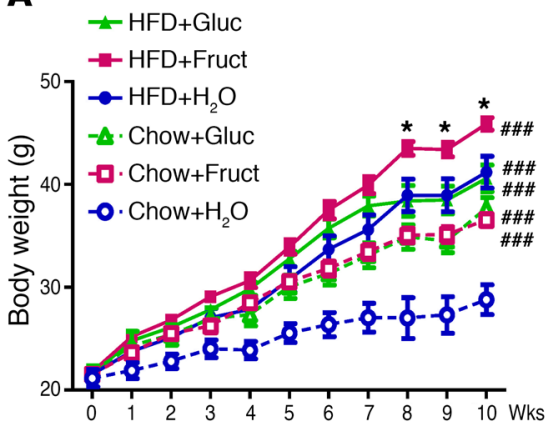

B

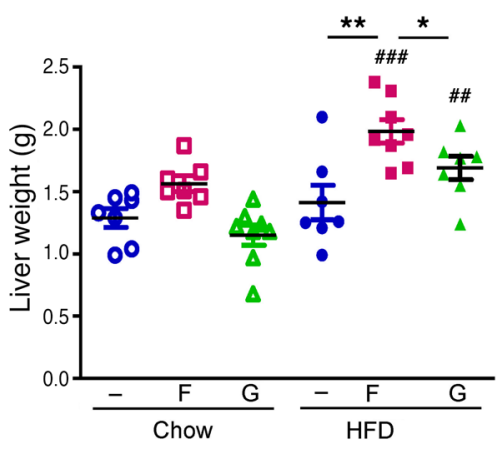

E

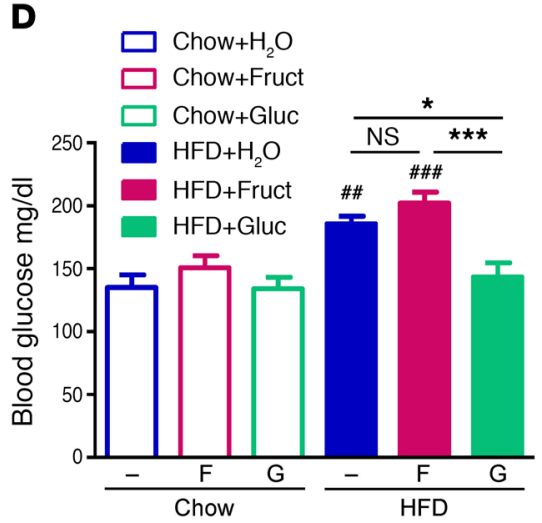

G

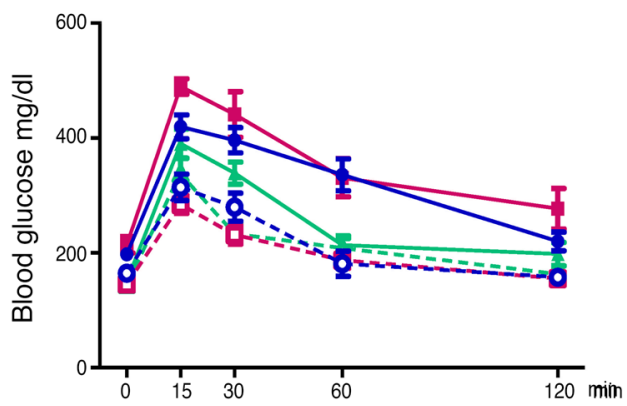

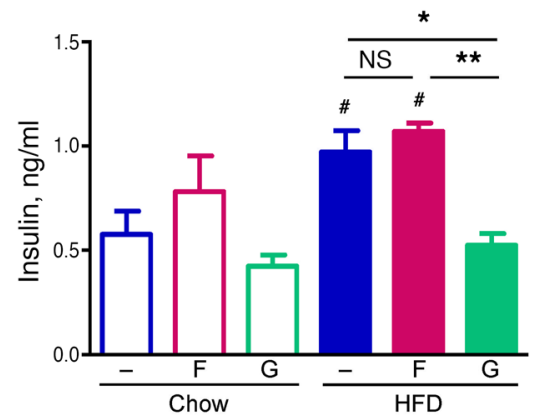

H
C

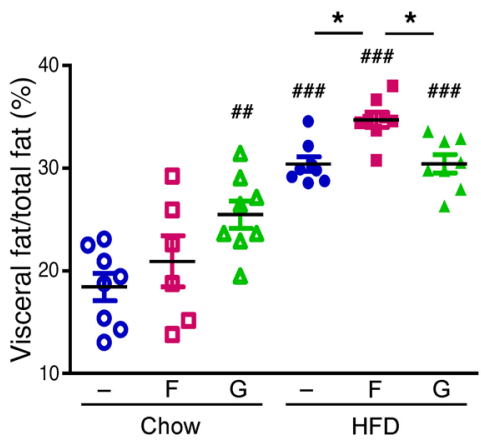

$\mathbf{F}$

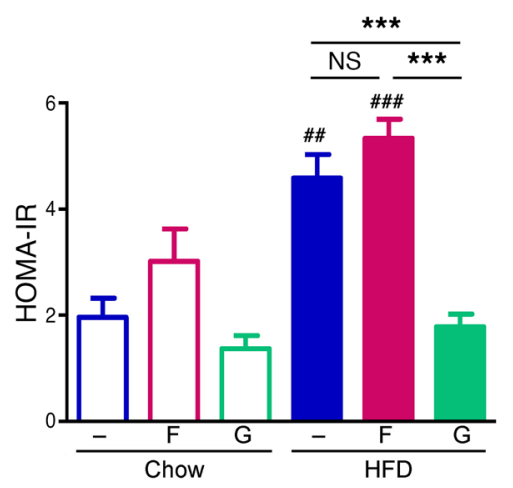

I
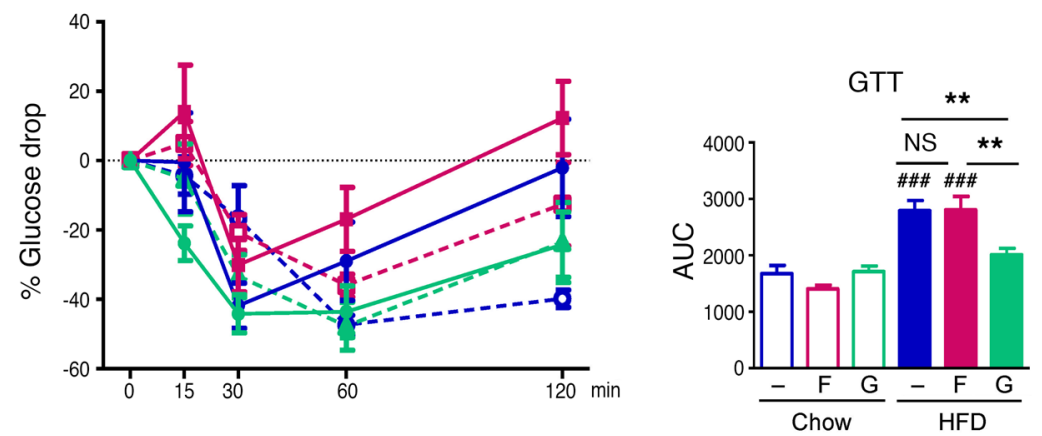

J

Chow : Fruct : Gluc : HFD : HFD+F : HFD+G
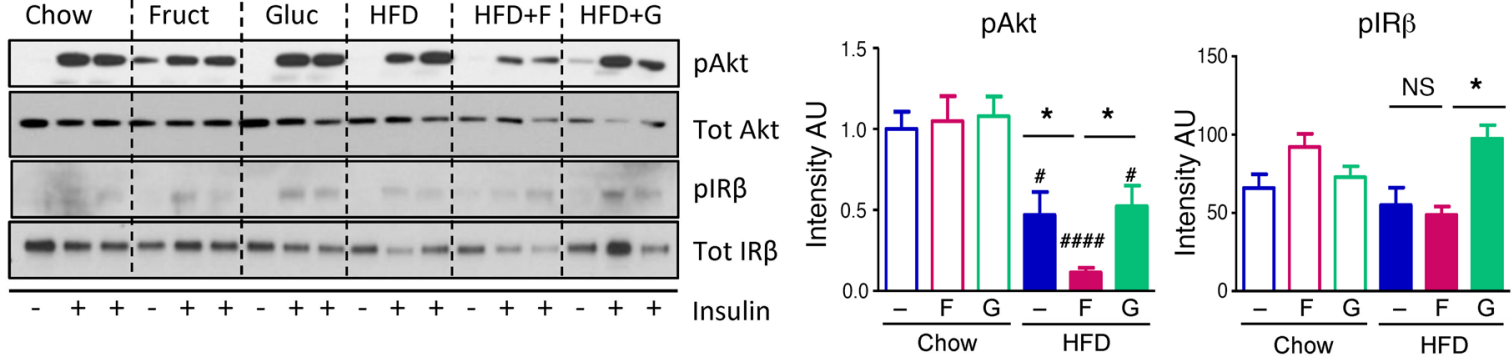

Figure 1. Fructose supplementation on HFD leads to higher weight gain and insulin resistance. (A) Weight gain of mice on chow and HFD, supplemented with either regular, $30 \%$ fructose, or glucose-sweetened water for 10 weeks. (B) Liver weights of the same mice at sacrifice. (C) Percentage of visceral fat/ total fat as measured by DEXA scan after 8 weeks on diet. (D) Blood glucose, (E) insulin levels and their calculated (F) HOMA-IR, measured after 8 weeks on diets. (G) Glucose tolerance test, (H) insulin tolerance test, and (I) glucose tolerance test calculated AUC measured after 8 weeks on diets. $n=7-8$ mice per group. (J) Western blot analysis and Image) quantification of insulin signaling in the liver. F, fructose; $G$, glucose. $n=6$ mice per group. Statistical analysis was performed using 2-way ANOVA with post hoc $t$ tests between the individual groups. ${ }^{\prime} P<0.05$; \#\# $P<0.01$; \#\# $P<0.001$; \#\#\# $P<0.0001$, compared with Chow $+\mathrm{H} 2 \mathrm{O}$ group. ${ }^{*} P<0.05 ;{ }^{* *} P<0.01$; ${ }^{* *} P<0.001$, within chow or HFD groups. 
hand, glucose has been reported to activate ChREBP by stimulating its expression, nuclear entry, and binding to its downstream targets (13). Recent evidence challenges this model by suggesting that fructose also activates ChREBP, specifically by inducing a second promoter in the ChREBP gene and an alternative splicing event to yield the ChREBP- $\beta$ isoform (14). It remains to be determined whether activation of different transcriptional programs by glucose and fructose affects hepatic lipid composition or simply the total amount of lipids.

Here, we show that consumption of fructose- versus glucosesweetened water in the presence of high-fat diet (HFD) leads to distinct metabolic phenotypes in mice. While both sugars similarly increase hepatic lipid accumulation, fructose supplementation on HFD is associated with increased expression of Srebp1c and Chrebp- $\beta$, increased fatty acid synthesis, and hepatic insulin resistance, while glucose supplementation on HFD is associated with increased total Chrebp and Chrebp- $\beta$ and liver triglyceride accumulation, but not with insulin resistance. We also found that ketohexokinase (KHK), the first enzyme of fructose metabolism, is increased both in mice supplemented with fructose and in obese adolescent humans with NAFLD. Suppression of KHK expression in the liver of fructose-fed mice leads to decreased expression of enzymes involved in fatty acid synthesis, better glucose tolerance, and improved NAFLD, suggesting that this may be a therapeutic target for treatment of NAFLD in humans.

\section{Results}

Consumption of fructose on HFD accelerates obesity and insulin resistance. Cohorts of 6-week-old male C57BL6/J mice were fed chow (21.6\% calories from fat) or HFD (60\% calories from fat) and given ad libitum access to drinking water or water containing 30\% (w/v) fructose or glucose. At the end of a 10-week study period, chow-fed mice supplemented with regular drinking water $\left(\right.$ Chow $\left.+\mathrm{H}_{2} \mathrm{O}\right)$ weighed $28.9 \pm 1.4 \mathrm{~g}$, whereas mice on chow diet supplemented with fructose (Chow+Fruct) or chow diet supplemented with glucose (Chow+Gluc) weighed $36.5 \pm 0.6 \mathrm{~g}$ and $37.7 \pm 1.0$ g, respectively (Figure $1 \mathrm{~A}$ ), both significantly more than the Chow $+\mathrm{H}_{2} \mathrm{O}$ group, consistent with their higher caloric intake (Supplemental Figure 1A; supplemental material available online with this article; https://doi.org/10.1172/JCI94585DS1). After 10 weeks on $\mathrm{HFD}$, mice on regular water $\left(\mathrm{HFD}+\mathrm{H}_{2} \mathrm{O}\right)$ weighed 41.2 $\pm 1.6 \mathrm{~g}$, significantly more $(P<0.001)$ than chow-fed controls, and those on HFD supplemented with fructose (HFD+Fruct) had the highest body weight $(45.9 \pm 0.6 \mathrm{~g}, P<0.05$ vs. HFD). Interestingly, mice on HFD supplemented with glucose (HFD+Gluc) did not gain additional weight $(40.6 \pm 1.3 \mathrm{~g})$ beyond the $\mathrm{HFD}+\mathrm{H}_{2} \mathrm{O}$ group, despite the fact that the estimated caloric intakes of the HFD+Fruct and HFD+Gluc mice were similarly increased compared with the $\mathrm{HFD}+\mathrm{H}_{2} \mathrm{O}$ group (Supplemental Figure 1A).

The additional weight gain in the HFD+Fruct group was in part accounted for by increased liver weight, which was significantly higher in the HFD+Fruct group than in the other HFD groups (Figure $1 \mathrm{~B}$ and Supplemental Figure 1B), and by increased percentage of visceral fat (Figure 1C). Liver weights of chow-fed mice supplemented with fructose or glucose were not different from control, but Chow+Gluc-fed mice did exhibit an increased percentage of visceral fat. In mice fed HFD, the percentage of body composition made up of subcutaneous fat was not affected by sugar-sweetened water intake and was actually decreased in all 3 groups compared with their chow-fed controls (Supplemental Figure 1C). There was no difference in lean body mass (Supplemental Figure 1D) or average spontaneous activity (Supplemental Figure 1E) in any groups of mice as compared with Chow $+\mathrm{H}_{2} \mathrm{O}$ control. Oxygen consumption $\left(\mathrm{VO}_{2}\right)$ of mice subjected to 24 hours of fasting followed by 48 hours of refeeding was higher in the dark phase for fasted, but not refed, mice supplemented with glucose, but not with fructose, on a chow diet. On HFD, $\mathrm{VO}_{2}$ was significantly lower in fasted and refed mice in the HFD+Fruct group, as compared with both the $\mathrm{HFD}+\mathrm{H}_{2} \mathrm{O}$ and $\mathrm{HFD}+\mathrm{Gluc}$ groups (Supplemental Figure 2A), contributing to increased weight gain in these mice. The respiratory exchange ratio (RER) was profoundly lower in Chow+Gluc mice compared with Chow $+\mathrm{H}_{2} \mathrm{O}$ and Chow + Fruct-fed mice, indicating higher rates of fatty acid oxidation (Supplemental Figure 2B). As expected, RER was depressed in all mice on HFD, abrogating the differences observed on a chow diet.

Despite consumption of high amounts of simple sugars, fructose or glucose supplementation did not affect fasting blood glucose in chow-fed animals (Figure 1D). Consumption of HFD significantly raised fasting blood glucose levels $(135 \pm 10 \mathrm{mg} / \mathrm{dl}$ for Chow $+\mathrm{H}_{2} \mathrm{O}$ vs. $186 \pm 6 \mathrm{mg} / \mathrm{dl}$ for $\mathrm{HFD}+\mathrm{H}_{2} \mathrm{O}$ group), and this was further increased in mice fed HFD+Fruct $(202 \pm 9 \mathrm{mg} / \mathrm{dl})$. Interestingly, blood glucose levels of HFD+Gluc mice $(143 \pm 11$ $\mathrm{mg} / \mathrm{dl}$ ) were lower than in both other HFD groups and were not different from those of chow-fed controls (Figure 1D). Fasting insulin levels were significantly elevated in the $\mathrm{HFD}+\mathrm{H}_{2} \mathrm{O}$ group, but were the highest in the HFD+Fruct group, as compared with the Chow $+\mathrm{H}_{2} \mathrm{O}$ group $(1.1 \pm 0.1 \mathrm{ng} / \mathrm{ml}$ vs $0.6 \pm 0.1 \mathrm{ng} / \mathrm{ml}$ ) (Figure 1E). Again, surprisingly, serum insulin levels of the HFD+Gluc group $(0.5 \pm 0.1 \mathrm{ng} / \mathrm{ml})$ were significantly lower than in both other HFD-fed groups and were not different from those of chow-fed controls, despite consumption of this major insulin secretagogue. Insulin resistance as estimated by HOMA-IR confirmed that both $\mathrm{HFD}+\mathrm{H}_{2} \mathrm{O}$ and $\mathrm{HFD}+$ Fruct groups were insulin resistant compared with chow-fed mice, while HFD+Gluc-fed mice had HOMA-IR values similar to those of the chow-fed groups (Figure $1 F)$. In line with these findings, oral glucose and insulin tolerance tests revealed that the HFD+Fruct group was the most glucoseintolerant and insulin-resistant cohort, but was not different from the $\mathrm{HFD}+\mathrm{H}_{2} \mathrm{O}$ cohort, while the HFD+Gluc group exhibited glucose tolerance and insulin sensitivity similar to those of chow-fed controls (Figure 1, G-I).

To further explore the effects of the various diets on insulin sensitivity, the activation of insulin signaling in the liver was evaluated 10 minutes following injection of $0.5 \mathrm{U}$ of insulin into the portal vein. Neither fructose nor glucose supplementation affected Akt phosphorylation on the chow diet; however, HFD decreased Akt phosphorylation by approximately 50\% (Figure 1J). Supplementation of HFD with fructose further decreased insulinstimulated Akt phosphorylation to approximately $10 \%$ of control levels, whereas supplementation with glucose had no effect beyond HFD alone. Autophosphorylation of the insulin receptor $\beta$ subunit (IR- $\beta$ ) was likewise impaired in mice on $\mathrm{HFD}+\mathrm{H}_{2} \mathrm{O}$ and HFD+Fruct diets, but interestingly, on HFD+Gluc diets, phosphorylation of IR- $\beta$ was similar to that in chow-fed controls (Fig- 
A
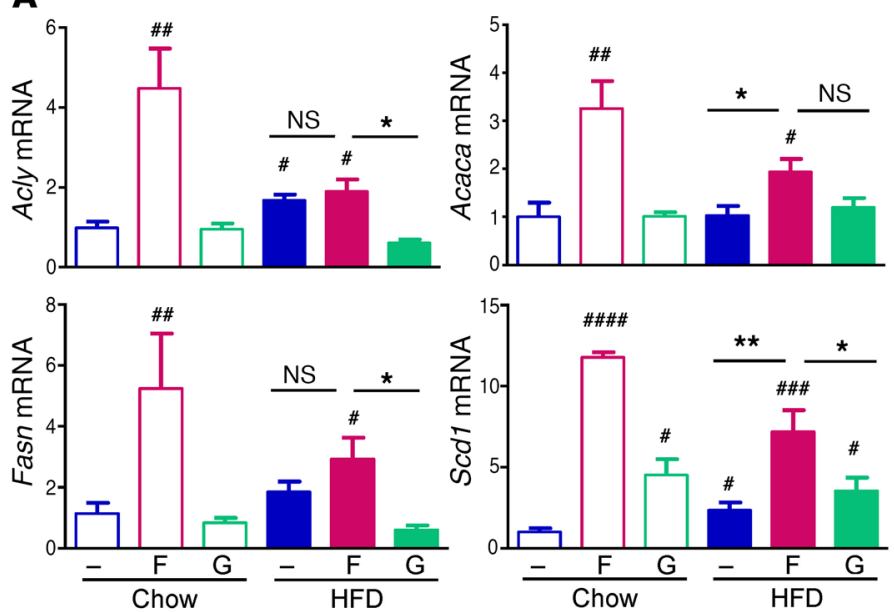

C

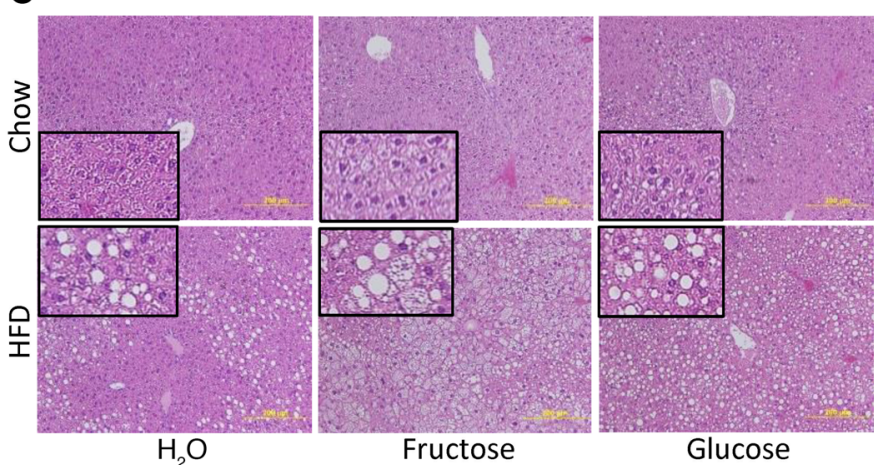

$\mathrm{H}_{2} \mathrm{O}$

E

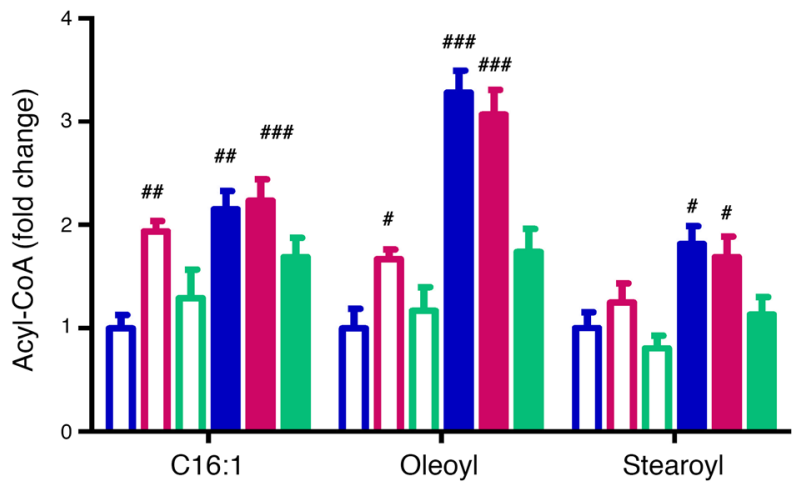

G

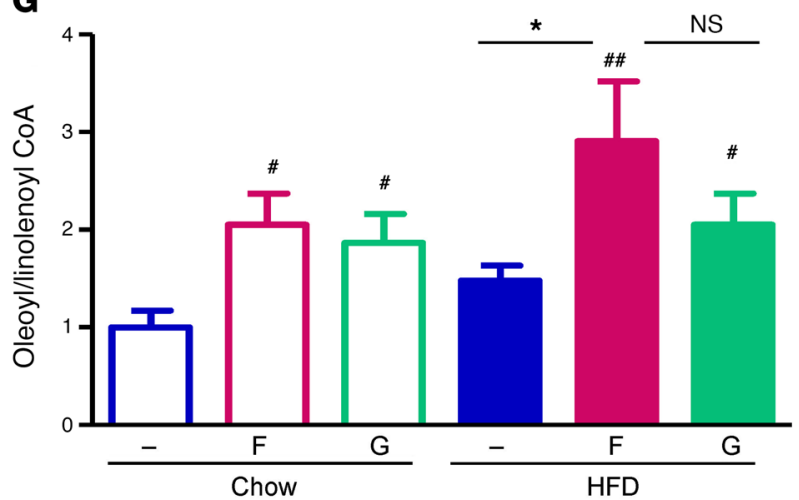

B
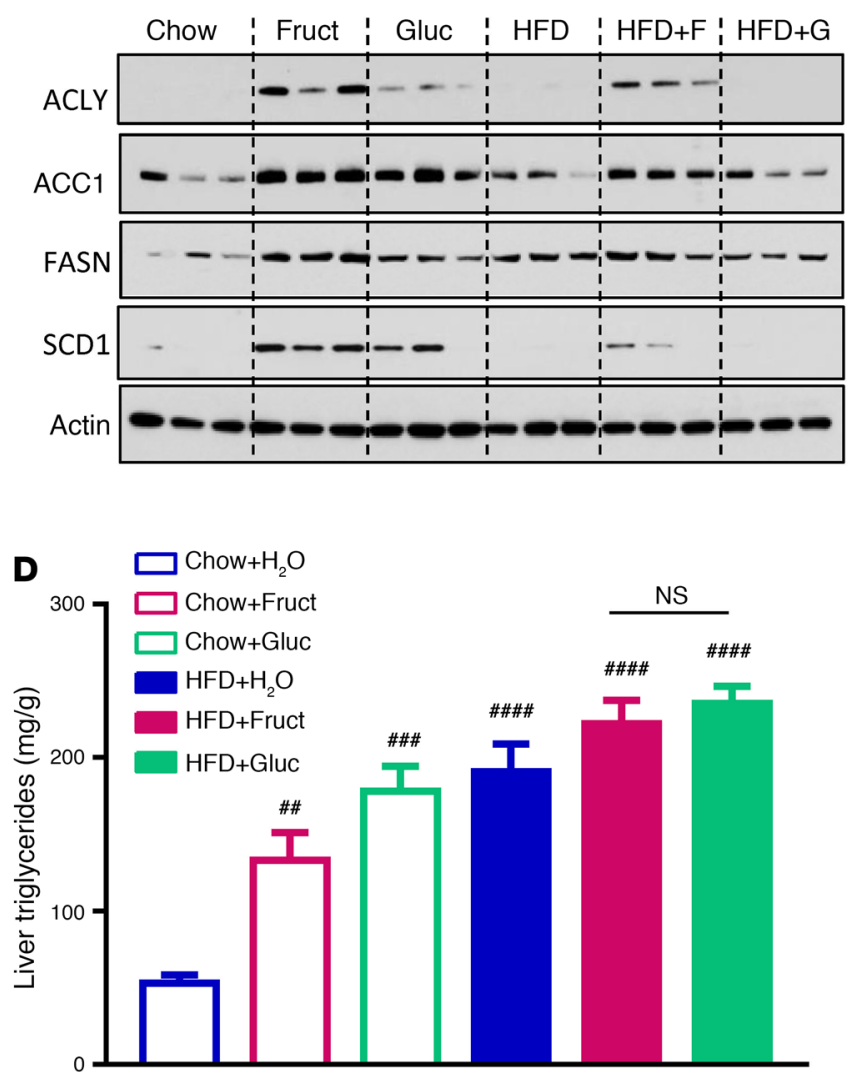

F
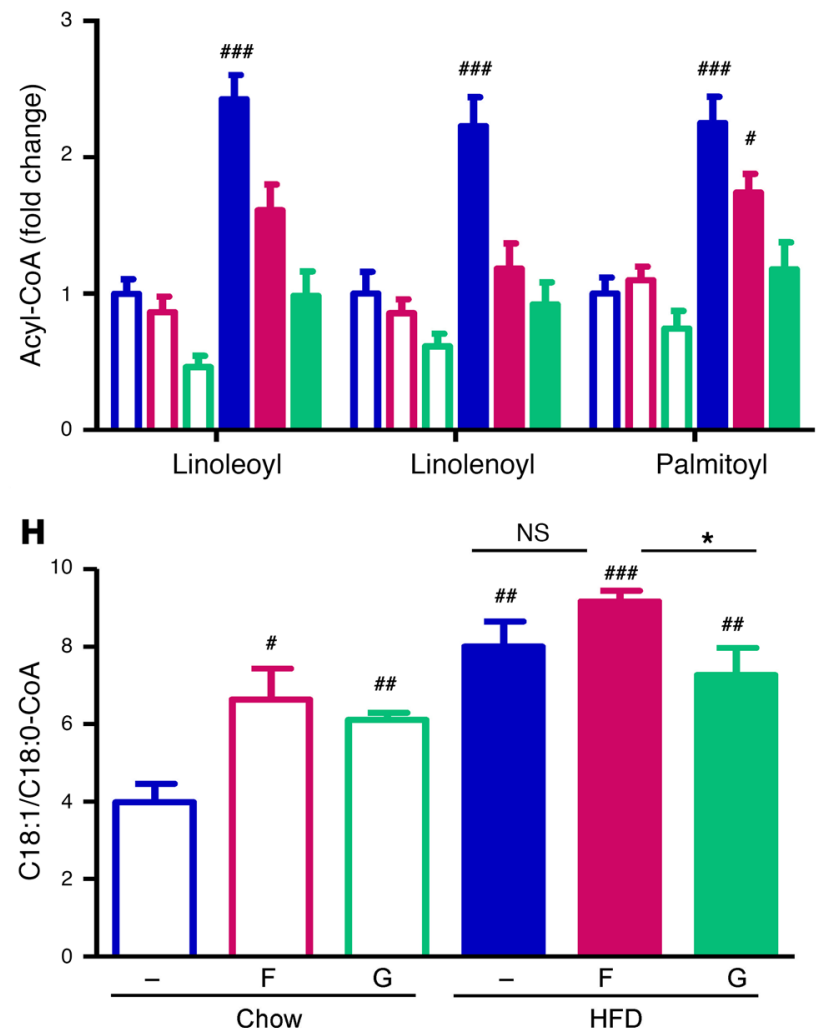
Figure 2. Fructose upregulates hepatic fatty acid synthesis. (A) mRNA expression and (B) protein levels of enzymes involved in fatty acid synthesis. (C) H\&E histology and (D) liver triglyceride content of mice after 10 weeks on different diets. Scale bars: $200 \mu \mathrm{m}$. Insert magnification, $\times 4 . n=7-8$ mice per group. LC/MS quantification of (E) endogenously synthesized and (F) exogenously ingested Acyl-CoAs. (C) The ratios of oleoyl-CoA to linolenoylCoA and $(\mathbf{H})$ C18:1-CoA to C18:0-CoA from livers of these mice. $n=6$ mice per group. Statistical analysis was performed using 2-way ANOVA with post hoc $t$ tests between the individual groups. ${ }^{\#} P<0.05$; ${ }^{\#} P<0.01$; ${ }^{\# \#} P<0.001$; \#\#\# $P<0.0001$, compared with Chow $+\mathrm{H}_{2} \mathrm{O}$ group. ${ }^{*} P<0.05$; ${ }^{* *} P<0.01$, within chow or HFD groups.

ure 1j). Furthermore, global tyrosine phosphorylation, in response to insulin, as assessed by anti-phosphotyrosine Western blotting, was also decreased in mice on $\mathrm{HFD}+\mathrm{H}_{2} \mathrm{O}$ and $\mathrm{HFD}+$ Fruct diets, but was unaffected in mice on an HFD+Gluc diet (Supplemental Figure 3A). While insulin-stimulated phosphorylation was decreased, basal IR- $\beta$ phosphorylation and basal Akt phosphorylation were increased with fructose feeding on HFD (Supplemental Figure 3B), likely reflecting the higher plasma insulin levels in the basal state, while the reduced insulin-induced phosphorylation reflected insulin resistance in fructose-supplemented mice. In contrast, basal Erk phosphorylation was decreased with fructose feeding on both chow and HFD (Supplemental Figure 3B).

Fructose and glucose consumption have unique effects on de novo lipogenesis. Fructose, but not glucose, supplementation in chowfed mice increased hepatic levels of mRNA-encoding enzymes that regulate fatty acid synthesis, including ATP citrate lyase $($ Acly), acetyl-CoA carboxylase $\alpha$ (Acaca), fatty acid synthase (Fasn), and stearoyl-CoA desaturase 1 ( $S c d 1$ ), by 3- to 12 -fold (Figure 2A). HFD alone did not affect the expression of these enzymes, but it blunted the effect of fructose. In agreement with the mRNA levels, protein levels of ACLY, ACC1, FASN, and SCD1 were increased 2- to 14-fold in mice on chow diet supplemented with fructose and to a lesser extent with glucose (Figure 2B). On HFD, protein levels of these enzymes also increased with fructose supplementation and were largely unchanged with glucose supplementation, while HFD had no detectable effect in increasing the protein levels of most of the lipogenic enzymes. Fructose or glucose supplementation for 10 weeks in chow-fed mice resulted in mild hepatic steatosis and increased triglyceride (TG) accumulation, as assessed by histology and measurement of triglycerides in liver homogenates (Figure 2, C and D). HFD alone induced even higher triglyceride accumulation and moderate steatosis, and this was further increased with sugar supplementation. Interestingly, the HFD+Fruct group developed severe steatosis with accumulation of both micro- and macrovesicular lipid droplets. In contrast, the HFD+Gluc group also developed severe steatosis, but in these mice, steatosis was almost exclusively composed of macrovesicular lipid droplets (Figure 2C).

Hepatic levels of fatty acyl CoAs, metabolic intermediates of de novo lipogenesis, were quantified using targeted mass spectrometry (MS) (15). Levels of C16:1-CoA, oleoyl-CoA, and steroyl-CoA, the most abundant endogenously synthesized acyl-CoAs, were increased by $50 \%$ to $90 \%$ with fructose, but not glucose, supplementation in mice on a chow diet (Figure 2E). These acyl-CoAs were also increased in the livers of mice in the $\mathrm{HFD}+\mathrm{H}_{2} \mathrm{O}$ and HFD+Fruct cohorts, but not in the HFD+Gluc cohort. In contrast, the levels of acyl CoAs derived from exogenously ingested essential fatty acids, such as linoleic and linolenic acid, and the most abundant fatty acid in rodent HFD, palmitic acid, were increased by HFD alone, but not by fructose supplementation of HFD (Figure $2 F$ ). In fact, supplementation of HFD with fructose, and especially with glucose, decreased these acyl-CoAs toward baseline levels. When considered together, the ratio of endogenously synthesized oleoyl-CoA to exogenously ingested linolenoyl-CoA was significantly elevated with fructose feeding on HFD (Figure 2G), indicative of increased fatty acid synthesis. Another marker of fatty acid synthesis and especially Scd1 activity is the ratio of monounsaturated to saturated fatty acids. Fructose feeding on chow and HFD was associated with increased ratio of monounsaturated C18:1 to saturated C18:0-CoA (Figure 2H). This desaturation index was increased in all HFD groups, but was significantly lower in the HFD+Gluc as compared with the HFD+Fruct group.

In addition to its effects on lipid metabolism, sugar supplementation had an effect on hepatic amino acid levels. Valine levels were reduced by $20 \%-40 \%$ with glucose, but not fructose, supplementation on chow and HFD, and a similar trend was observed for leucine/isoleucine (Supplemental Figure 4A). The lower levels of these branched-chain amino acids could contribute to improved insulin sensitivity of glucose-supplemented mice (16). The levels of essential amino acids methionine, phenylalanine, and histidine were also markedly lower in HFD-fed mice, but were unaffected by sugar supplementation, and the levels of the conditionally essential amino acids tyrosine, glycine, and proline followed a similar trend. Interestingly, the hepatic levels of nonessential amino acids glutamine/glutamic acid, arginine, and citrulline were higher with glucose, but not fructose, supplementation of chow diet, and the levels of alanine, asparagine/aspartic acid, and serine were also higher with glucose supplementation on a chow diet, but the differences did not reach statistical significance. Mice consuming HFD generally had decreased levels of nonessential amino acids. In summary, fructose supplementation and HFD feeding resulted in decreased levels of the majority of hepatic amino acids, whereas glucose supplementation was associated with decreased levels of branched chain amino acids, but increased levels of nonessential, endogenously synthesized amino acids. TCA cycle intermediates in the liver were not significantly altered by fructose or glucose supplementation, with the exception of citrate, which was increased in the Chow+Gluc group and all HFD groups (Supplemental Figure 4B).

Fructose and glucose favorably upregulate distinct lipogenic transcription factors. Two major transcriptional regulators of hepatic lipogenesis are SREBP1c and ChREBP (11-13). Fructose supplementation of chow-fed mice led to an almost 3-fold increase in the expression of Srebp1c, whereas glucose supplementation was associated with a modest decrease in Srebp1c. A similar pattern was observed in mice on HFD, but as with the expression of lipogenic enzymes shown above (Figure 2C), the magnitude of the effect was blunted (Figure 3A). SREBP1 protein levels were not changed with fructose supplementation, whereas glucose decreased SREBP1 protein on both chow and HFD (Figure 3B). The activity of SREBP1c is regulated by both its protein level and posttranslational modifications. SREBP1c is made in the ER as a $125-\mathrm{kDa}$ precursor protein (P-SREBP1), 
A



C
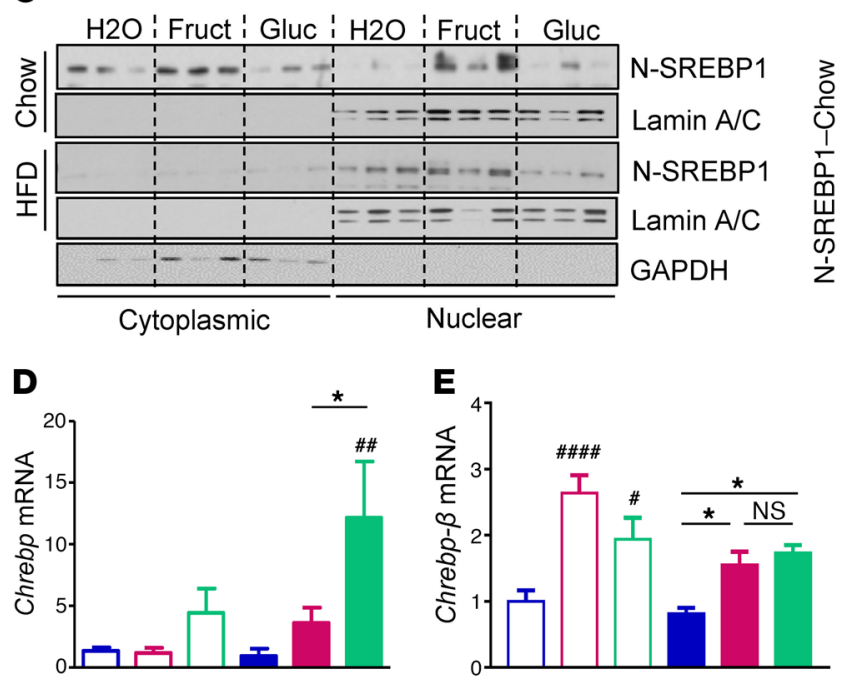

G

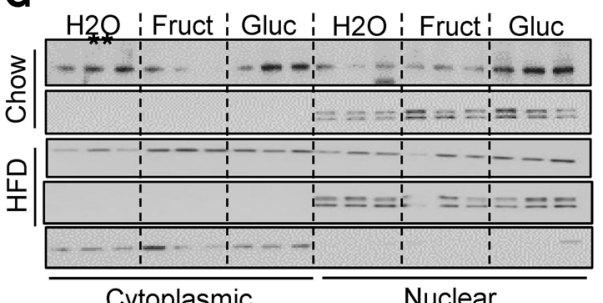

B

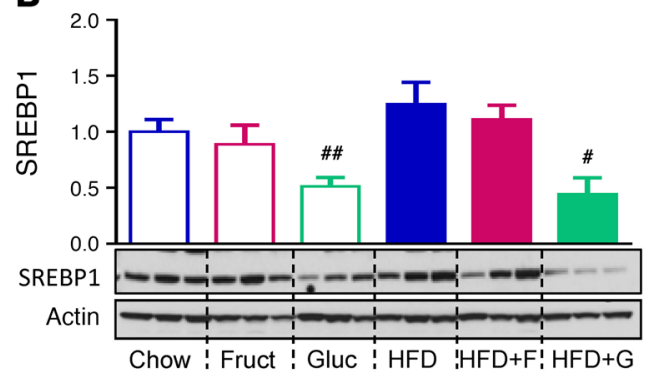

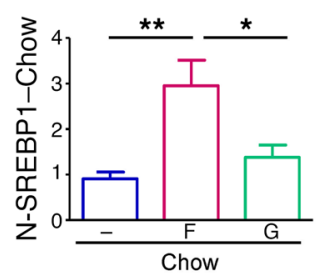

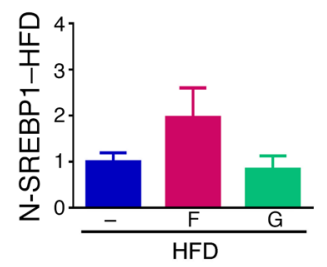

$\mathbf{F}$

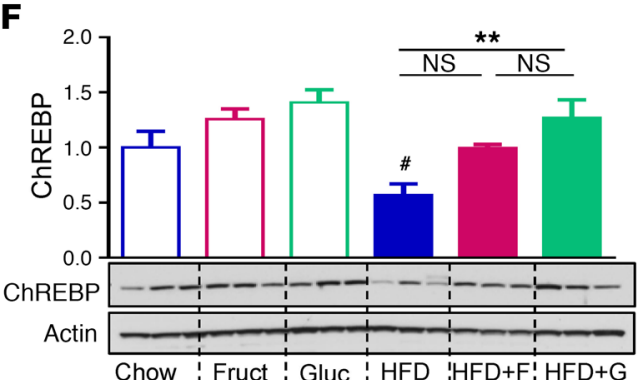

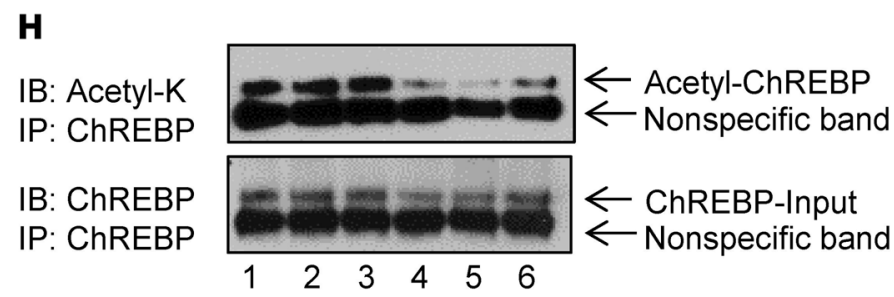

Figure 3. Fructose and glucose induce unique lipogenic transcription factors. (A) mRNA expression and (B) protein levels of SREBP1 transcription factors in whole cell lysates from livers of mice after 10 weeks on diets. (C) Western blots of cytoplasmic and nuclear fractions of truncated active form of (N) SREBP1 and Imagel quantification of N-SREBP1 protein. (D) mRNA expression of total Chrebp, (E) Chrebp- $\beta$ isoform, and (F) total protein levels of ChREBP in whole cell liver lysates. (C) Western blots of cytoplasmic and nuclear factions of ChREBP and Imagel quantification of nuclear fraction. $n=6$ mice per group. (H) Immunoprecipitation of ChREBP followed by immunoblot for acetyl-K. 1, Chow+ $\mathrm{H}_{2} \mathrm{O} ; 2$, Chow+Fruct; 3, Chow+Cluc; 4, HFD+H $\mathrm{O} ; 5, \mathrm{HFD}+\mathrm{Fruct} ; 6$, HFD+Gluc, with 3 samples pooled per group. Statistical analysis was performed using 2-way ANOVA with post hoc $t$ tests between the individual groups.

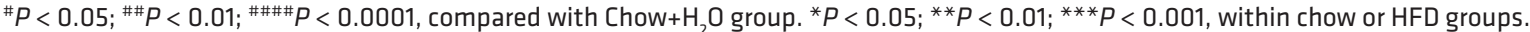

which is transported into the Golgi, where it is cleaved to an active $68-\mathrm{kDa} \mathrm{N}$-terminal fragment $(\mathrm{N}-\mathrm{SREBP} 1 \mathrm{c})$ that is transported into the nucleus to regulate gene expression (17). Fructose supplementation increased the levels of cleaved N-SREBP1 nuclear protein in the liver by 3 -fold in chow-fed mice and by 2-fold in HFD-fed mice, while glucose supplementation had no effect on either diet (Figure 3C).

By comparison, glucose supplementation of chow-fed mice increased total Chrebp mRNA levels by 4 -fold, and this was doubled again in mice on HFD+Gluc supplementation. Fructose had 
no effect on total Chrebp mRNA in chow-fed mice, but did produce a modest increase in HFD-fed mice, only one-third as much as observed in HFD+Gluc-fed mice (Figure 3D). Chrebp exists as both $\alpha$ - and $\beta$-isoforms due to alternative promoter use and alternative splicing, and the $\beta$-isoform has been reported to be regulated by fructose $(14,18)$. Consistent with this, we found that both fructose and glucose supplementation of chow-fed mice increased the expression of Chrebp- $\beta$ by 2- to 3-fold, an effect that was attenuated in mice on HFD (Figure 3E). One downstream target of ChREBP is liver-pyruvate kinase $(l-P k)$, and its expression closely mimicked that of Chrebp- $\beta$ (Supplemental Figure 5A). Total ChREBP protein levels were assessed by immunoblotting with an antibody that recognizes both ChREBP isoforms. Total ChREBP protein levels measured with this antibody were reflective of total Chrebp mRNA expression, with both showing a tendency to be higher in chow- and HFD-fed mice on glucose supplementation (Figure 3F). Nuclear translocation of ChREBP was also affected by sugar supplementation such that glucose, but not fructose, increased nuclear levels of ChREBP by 3-fold in mice on normal chow, and this effect was again attenuated in mice on HFD (Figure 3G). Activity of ChREBP is also regulated by acetylation on lysine 672 (19), and this was decreased in the $\mathrm{HFD}+\mathrm{H}_{2} \mathrm{O}$ and HFD+Fruct groups as compared with the control, while acetylation of ChREBP was increased in the HFD+Gluc group as compared with the other HFD-fed mice (Figure $3 \mathrm{H}$ ).

Glucose and fructose supplementation regulate different gene sets. Global gene expression in the liver was assessed by RNA-sequence (RNA-seq) analysis after 10 weeks on chow, HFD, or HFD supplemented with glucose or fructose. Principal component analysis of the gene expression data revealed that, compared with a relatively modest effect of HFD as compared with chow, addition of glucose or fructose to HFD induced major changes in gene expression, with the greatest effect being that of glucose (Figure 4A). Volcano plot analysis of the HFD+Gluc versus HFD+Fruct data identified genes involved in fatty acid synthesis, such as Fasn, Acly, and Acaca, as the most significantly upregulated genes by fructose supplementation, whereas the most significantly upregulated genes by glucose supplementation were the genes regulating triglyceride synthesis, such as glycerol 3-phosphate acyltransferase (Agpt9) and the genes regulating fatty acid oxidation, such as carnitine transporter solute carrier family 22, member 5 (Slc22a5), and acylCoA thioesterase 1 (Acot1) (Figure 4B).

The hepatic de novo lipogenesis pathway was uniquely affected by fructose and glucose supplementation, so that a part of the pathway upregulated in the HFD+Fruct, but not the HFD+Gluc, group contained the above-mentioned most significantly upregulated genes as well as other genes involved in fatty acid synthesis, such as $S c d 1$, pyruvate dehydrogenase kinase, isoenzyme 3 (Pdk3), and elongation of long chain fatty acids member 5 (Elovl5) and member 6 (Elovl6) (Figure 4C). Furthermore, many SREBP1c targets, in addition to fatty acid synthesis genes, such as malic enzyme $1(M e 1)$ and hexokinase $2(H k 2)$, were increased in mice fed an HFD+Fruct, but not an HFD+Gluc, diet. In contrast, genes upregulated in HFD+Gluc, but not HFD+Fruct, included genes involved in triglyceride synthesis, such as Agpat1, Agpat9, the glycerol-3-phosphate transporter, member 1 (Slc37a1), and diacylglycerol o-acyltransferase 2 (Dgat2) (Figure 4C). Taken together, these data show that fructose supplementation enhanced expres- sion of fatty acid synthesis genes, while glucose supplementation upregulated genes involved in triglyceride synthesis.

ChREBP has many overlapping functions with SREBP1c, as both have been reported to increase expression of lipogenic genes, such as Pklr, Fasn, and Acaca (20). However, genes involved in lipid oxidation, such as fibroblast growth factor 21 (Fgf21) (21) and carnitine palmitoyltransferase 1 (Cpt1a) (22), have only been reported to be induced by ChREBP. Indeed the expression of these genes as well as many other genes involved in fatty acid oxidation, such as carnitine palmitoyltransferase 2 (Cpt2); family member 12, medium, long, and very long chain acyl-coenzyme A dehydrogenase (Acad12, Acadm, Acadl, Acadvl); acetyl-coenzyme A acyltransferase 2 (Acaa2), and the $\alpha$ and $\beta$ subunits of hydroxyacylcoenzyme A dehydrogenase (Hadha and Hadhb), were increased in HFD+Gluc, but not in the HFD+Fruct group (Figure 4D), confirming that many ChREBP targets were increased with glucose supplementation as compared with the downstream targets of SREBP1c, which were increased with fructose.

Striking differences in hepatic gene expression were seen for mRNAs coding for proteins involved in the insulin signaling pathway in the HFD and fructose- versus glucose-supplemented groups (Figure 4E). HFD alone increased the expression of glucokinase $(G c k)$ and phosphatidylinositol 3-kinase, p85a subunit (Pi3kr1); HFD+Fruct induced phosphatidylinositol 3-kinase, $\mathrm{p} 110 \alpha$ subunit ( $\mathrm{Pik}$ Ca) and mitogen-activated protein kinase 9 (Mapk9), whereas HFD+Gluc induced the insulin receptor (Insr) and Akt1 mRNA. Increases in Pi3kr1 (23) and Mapk9 (24) have been observed in insulin-resistant states, while increases in the insulin receptor in the glucose-supplemented group might lead to improved insulin action (25). These changes in gene expression could explain, in part, the insulin resistance in HFD and HFD+Fruct mice versus the improved insulin signaling in HFD+Gluc mice.

Other remarkable differences in gene expression, however, were observed in mRNAs coding for proteins involved in mitochondrial function (Figure 4F). As compared with chow diet, the mice in the HFD-alone group had upregulation of mRNA coding for mitochondrial chaperons (heat shock protein 5 [Hspa5], heat shock protein 90, alpha family class b, member 1 [Hsp90ab1], chaperonin containing Tcp1, subunit 7 [Cct7]) and increases in genes regulating reactive oxygen species, such as reactive oxygen species modulator 1 (Romo1) and superoxide dismutase 1 (Sod1), whereas genes regulating fatty acid transport, such as solute carrier family 27, member 2 (Slc27a2), and fat storage-inducing transmembrane protein 2 (Fitm2), were decreased in mice on HFD. Addition of fructose to HFD induced unique changes in the expression of these mitochondrial genes, whereas glucose supplementation reversed the effect of both upregulated and downregulated genes in HFD-fed mice.

KHK is overexpressed in fructose-supplemented mice and in patients with NASH. KHK catalyzes the first step of intracellular fructose metabolism (26). Khk expression was increased 2-fold in mice supplemented with fructose on a chow diet and 3-fold in mice supplemented with fructose on an HFD, as assessed by quantitative PCR (qPCR) (Figure 5A) or RNA-seq (Figure 4C). Glucose supplementation or HFD itself did not significantly elevate $K h k$ expression (Figure $5 \mathrm{~A}$ ).

To investigate whether this might also be true in humans, liver biopsy samples were obtained from 12 obese adolescent patients 
A

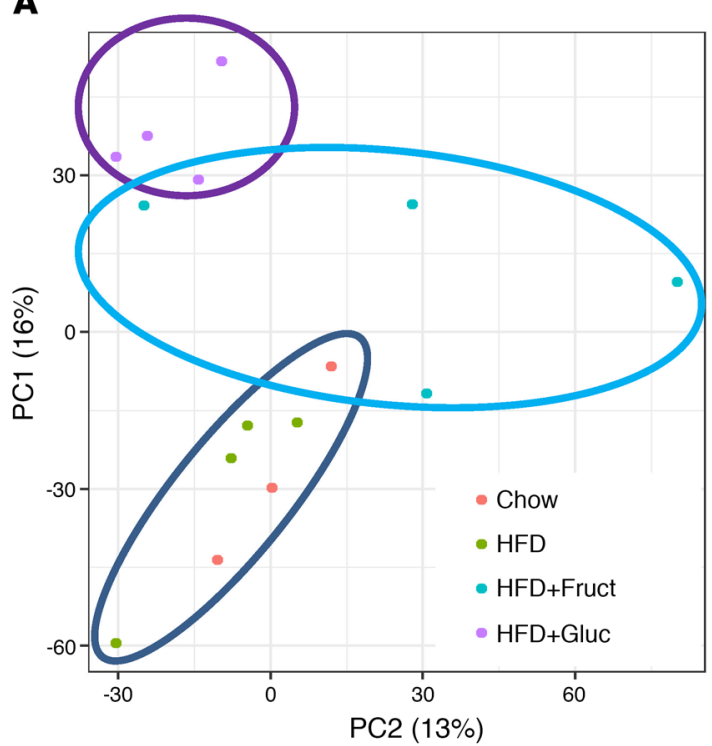

C

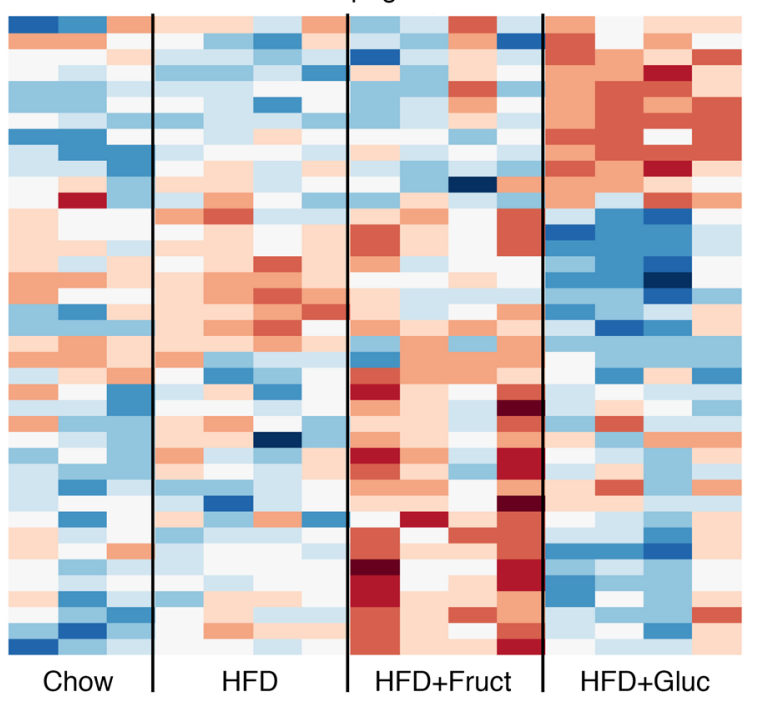

$\mathbf{E}$

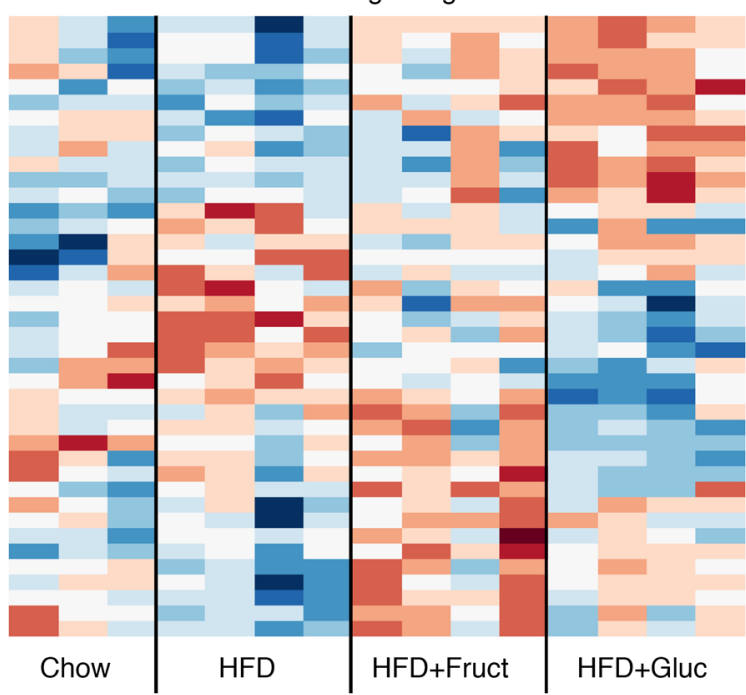

B

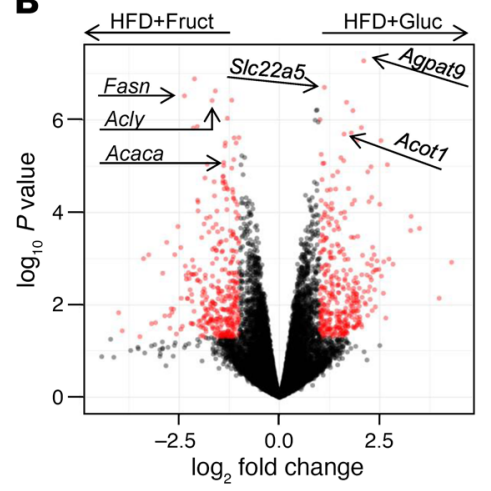

Figure 4. Hepatic gene expression induced by HFD and sugar metabolism.

(A) Principal component analysis of RNAseq data from the livers of mice following 10 weeks of diets. PC1, principal component 1. (B) Volcano plot comparison of genes induced in $\mathrm{HFD}+\mathrm{Cluc}$ versus $\mathrm{HFD+-}$ Fruct groups. Heatmap representation of genes involved in (C) de novo lipogenesis, (D) fatty acid metabolism, (E) insulin signaling, and (F) mitochondrial function pathways. $n=3-4$ samples per group.
D

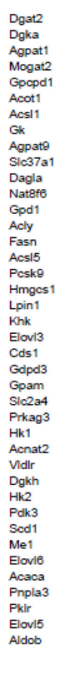

Fatty acid metabolism

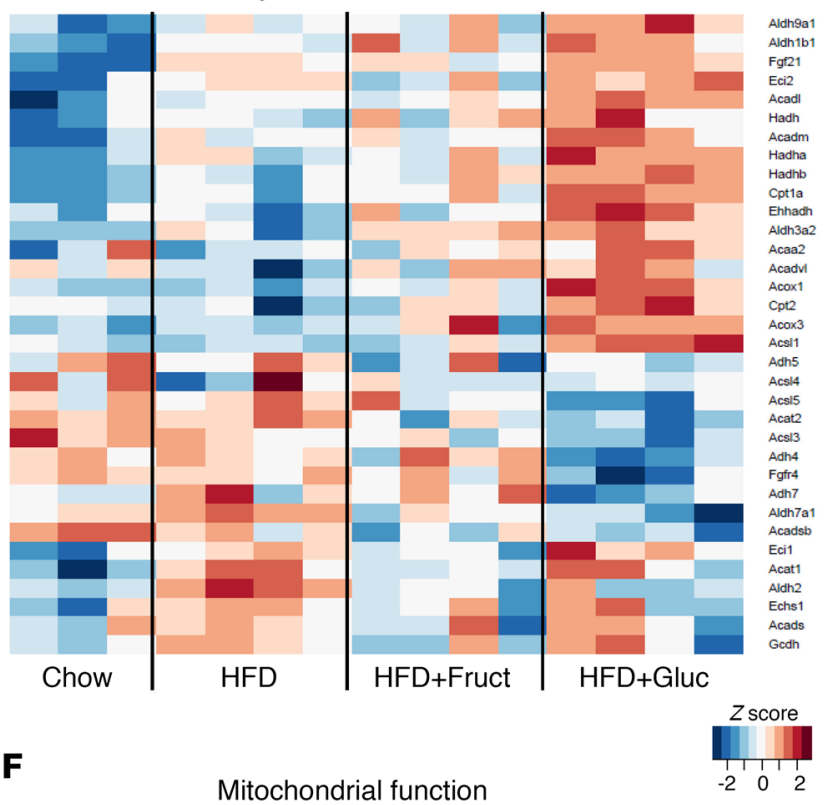


undergoing bariatric surgery (Supplemental Figure 5B). While all patients were obese, liver biopsy revealed that 4 had no extra fat accumulation in the liver (NoFL), 4 had simple steatosis and 4 had steatosis with inflammation (nonalcoholic steatohepatitis [NASH]). Similar to the changes observed in mice, KHK expression was elevated 2-fold in obese patients with more advanced liver disease as compared with obese subjects without fatty liver (Figure 5B). Mirroring the increase in mRNA, KHK protein levels were also increased 2-fold in NASH patients as compared with both NoFL and steatosis groups. Liver transcripts encoding lipogenic enzymes, such as ACLY, ACACA, FASN, and SCD1, were also increased 2- to 10-fold in liver samples from the NASH group (Figure 5C). In agreement with these results, protein levels of these enzymes were elevated in the NASH group as compared with the NoFL group (Figures 5, D and E).

Knockdown of KHK improves liver steatosis. To assess the role of KHK in the negative metabolic effects associated with fructose consumption, we knocked down the expression of $K h k$ in the liver at the mRNA level by biweekly subcutaneous injections of Gal-Nac-conjugated liver-specific siRNA in C57BL/6 mice that had been on HFD for 6 weeks and provided with either regular or fructose- or glucose-supplemented water. After 4 weeks of siRNA treatment, while maintained on the same diet, mRNA levels of $K h k$ were decreased by more than $90 \%$ in all 3 groups, and this was confirmed at the protein level (Figure 6A). At the end of 10 weeks, the HFD+Fruct cohort treated with KHK siRNA gained significantly less weight $(5.9 \pm 0.4 \mathrm{~g})$ than mice treated with control siRNA $\left(7.4 \pm 0.3 \mathrm{~g}\right.$ ) (Figure 6B), while mice on $\mathrm{HFD}+\mathrm{H}_{2} \mathrm{O}$ or HFD+Gluc gained an amount of weight similar to that of mice treated with a control siRNA. A major fraction of the difference in weight gain was a difference in liver weight, which was decreased by $30 \%$ in the HFD+Fruct group treated with KHK siRNA as compared with the control siRNA (Figure 6C). Somewhat surprisingly, liver weight was also markedly decreased with $K h k$ knockdown in the $\mathrm{HFD}+\mathrm{H}_{2} \mathrm{O}$ group and also tended to be lower in HFD+Gluc, but the latter did not reach significance. Reflecting the decrease in liver weight, liver triglyceride content was decreased $30 \%-35 \%$ in all groups treated with KHK siRNA (Figure 6D). This decrease in liver TG was confirmed by histology showing improved hepatic steatosis in all 3 groups treated with KHK siRNA as compared with the control groups (Figure 6E). Hepatic glycogen content tended to be slightly higher following KHK knockdown based on histologic assessment, while glycogen content was not different among mice on different diets (Supplemental Figure 6A). The severity of other NASH features, e.g., inflammation, ballooning degeneration, and fibrosis, was minimal in all groups of mice at 10 weeks on diets and was not affected by KHK siRNA administration. NAFLD activity score (NAS) was assessed as previously published (27), and it was not different among HFD-fed mice provided with either regular $(4.3 \pm 0.3)$ or fructose- $(4.0 \pm 0.6)$ or glucose-supplemented water $(4.5 \pm 0.3)$, but it decreased in all groups following knockdown of $\operatorname{KHK}(3.0 \pm 0.4,2.7 \pm 0.3$, and 3.8 \pm 0.6$)$ (Supplemental Figure 6B), and this was primarily driven by improvements in steatosis. Due to a small sample size of individual groups, all mice treated with control siRNA were combined into one group and compared with all mice treated with KHK siRNA. Interestingly, knockdown of Khk in all mice, irrespective of sugar supplementation, resulted in signifi- cantly decreased NAS as compared with in the combined controls (4.3 \pm 0.2 vs. $3.2 \pm 0.3 ; P<0.01$ ) (Supplemental Figure 6C).

As shown above, mRNA expression of Acly, Acaca, Fasn, and $S c d 1$ was elevated in livers of mice in the HFD+Fruct group as compared with the $\mathrm{HFD}+\mathrm{H}_{2} \mathrm{O}$ and HFD+Gluc groups, and the expression of all 4 of these enzymes decreased by $30 \%-65 \%$ in the HFD+Fruct group following Khk knockdown (Figure 6F). Similarly, protein levels of ACLY, ACC1, and FASN were increased in the HFD+Fruct group and decreased following Khk knockdown (Figure 6G), consistent with the mRNA data. Interestingly, mRNA expression and protein levels of these enzymes were largely unchanged in the HFD and HFD+Gluc groups following $K h k$ knockdown, indicating that the improvement in steatosis in these 2 groups was not secondary to the effects of $K h k$ on fatty acid synthesis, but most likely was secondary to the effects on other processes, such as mitochondrial function or fatty acid metabolism, which is the subject of our follow-up study.

KHK knockdown improves glucose tolerance. In agreement with improved liver steatosis, glucose tolerance improved in the HFD+Fruct group treated with $K h k$, as compared with control siRNA (Figure 7A). Glucose tolerance also improved in the HFD+Gluc group following Khk knockdown at 30 and 60 minutes after oral glucose administration, and it tended to be lower in the $\mathrm{HFD}+\mathrm{H}_{2} \mathrm{O}$ group treated with KHK siRNA, but the latter did not reach statistical significance (Figure 7B). As previously shown, insulinstimulated Akt phosphorylation was decreased by $80 \%$ in the HFD+Fruct group as compared with all other HFD-fed groups, and it improved to baseline level following knockdown of $K h k$ (Figure 7, C and D). Akt phosphorylation in the HFD+Gluc group also increased with Khk knockdown and tended to be increased in the $\mathrm{HFD}+\mathrm{H}_{2} \mathrm{O}$ group. The improvement in glucose tolerance was not a result of decreased adipose tissue mass, as the weight of subcutaneous, perigonadal, and brown adipose tissue did not change in the HFD and HFD+Fruct groups following Khk inhibition; in fact, the weights of all 3 adipose tissue depots increased in the HFD+Gluc group following Khk knockdown (Supplemental Figure 7A). Furthermore, there were no deleterious effects of $K h k$ knockdown in liver on kidney function, as BUN and creatinine (Supplemental Figure 7B) did not increase following knockdown, although it is likely that more fructose is available to be metabolized in the kidney, which also has high Khk expression.

\section{Discussion}

In the present study, we have explored the interaction between the dietary sugars fructose and glucose, both on chow and HFD, on the pathogenesis of fatty liver disease and insulin resistance and investigated the mechanisms underlying these effects. While both fructose and glucose supplementation on chow diet results in increased weight gain and mild liver steatosis as compared with regular water, this does not lead to insulin resistance. On HFD, we found more profound metabolic differences, such that HFDfed mice supplemented with fructose in drinking water developed more pronounced obesity, glucose intolerance, and impaired insulin signaling as compared with mice on HFD supplemented with equal amounts of glucose. Whereas both sugars increased total hepatic lipid accumulation, interestingly, fructose in particular increased fatty acid synthesis, while glucose preferentially 
A
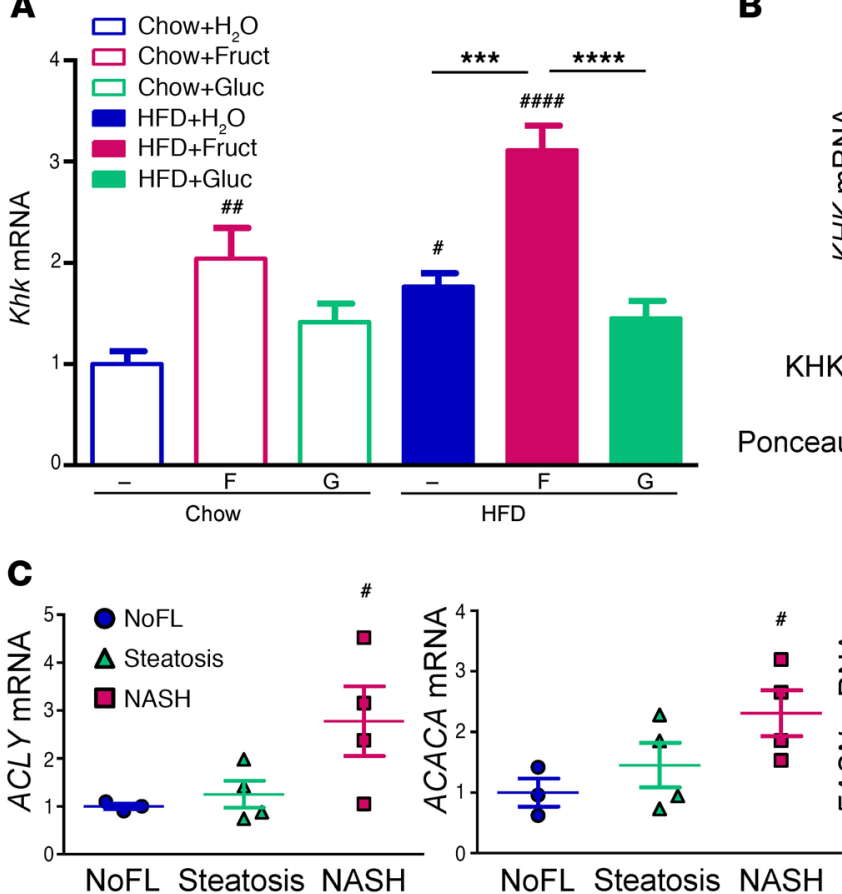

B

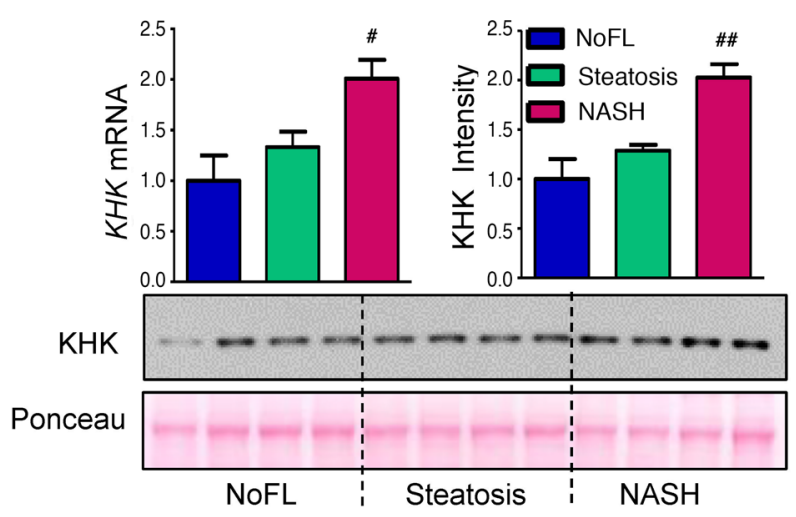

D

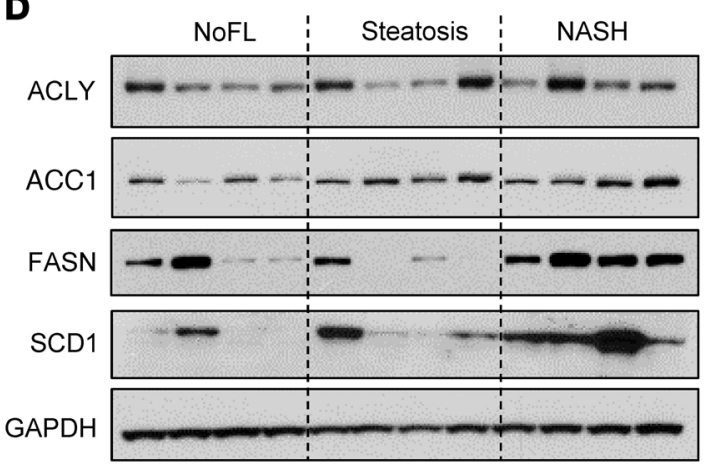

E

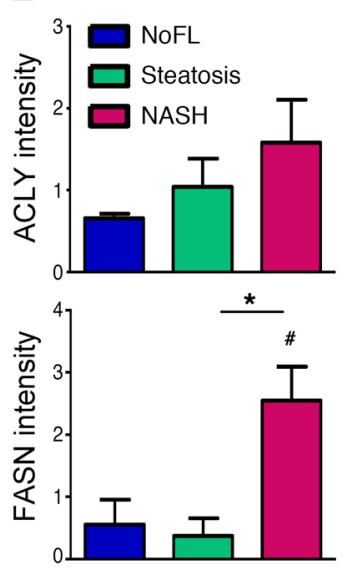

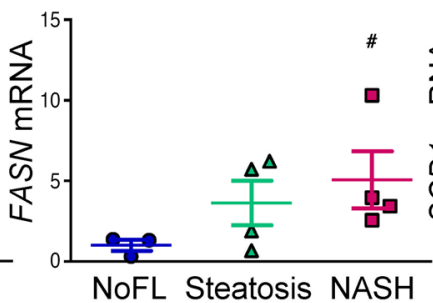

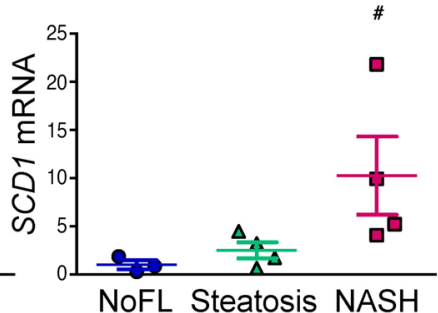

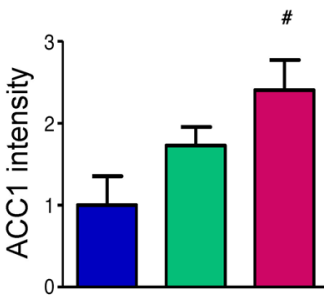

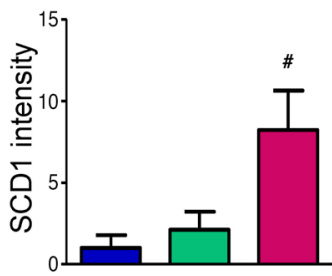

Figure 5. KHK is induced with fructose supplementation and in patients with progressive liver disease. (A) mRNA expression of $K h k$ in the livers of mice at 10 weeks on different diets. $n=6$ mice per group. Statistical analysis was performed using 2-way ANOVA with post hoc $t$ tests between the individual groups. ${ }^{\#} P<0.05$; ${ }^{\# \#} P<0.01$; \#\#\#\# $<0.0001$, compared with Chow $+\mathrm{H}_{2} \mathrm{O}$ group. ${ }^{* * *} P<0.001$; ${ }^{* * * *} P<0.0001$, within chow or HFD groups. (B) KHK mRNA and protein levels in the livers of obese adolescent patients undergoing bariatric surgery. (C) mRNA expression of enzymes regulating fatty acid synthesis as well as (D) Western blot analysis and (E) Imagel quantification of their protein levels. $n=4$ subjects per group. Statistical analysis was performed using 1-way ANOVA. $\# P<0.05 ;{ }^{\#} P<0.01$, compared with NoFL group. ${ }^{*} P<0.05$, between steatosis and NASH groups.

increased triglyceride synthesis. Furthermore, knockdown of fructose metabolism led to improved liver steatosis and insulin resistance. These results indicate that the fructose component of dietary sugar is uniquely associated with poor metabolic outcomes, whereas glucose supplementation on HFD, surprisingly, has beneficial metabolic effects.

While many studies have documented poor metabolic outcomes associated with increased consumption of dietary sugar in humans $(4,5,28)$, it is unclear what downstream pathways mediate this process. Gene expression profiling reveals that, while both sugars can induce Chrebp- $\beta$ isoform, fructose also specifically increased the expression of Srebp1c and its downstream targets, the enzymes regulating fatty acid synthesis, whereas glucose increased expression of total Chrebp and Chrebp- $\beta$ and enzymes regulating triglyceride synthesis. These findings are supported by previous studies evaluating each sugar independently, which have shown that fructose can induce hepatic expression of Srebp1c (12) and Chrebp- $\beta$ (14) and that glucose can induce total Chrebp and Chrebp- $\beta$ expression $(13,14)$. Similar findings have been suggested by human studies that have found elevated levels of SREBP1 in patients with fatty liver disease (29), while increased total ChREBP expression has been observed in states in which hepatic steatosis is dissociated from insulin resistance (30). Likely a degree of overlap of gene regulation exists between these transcription factors, 
A

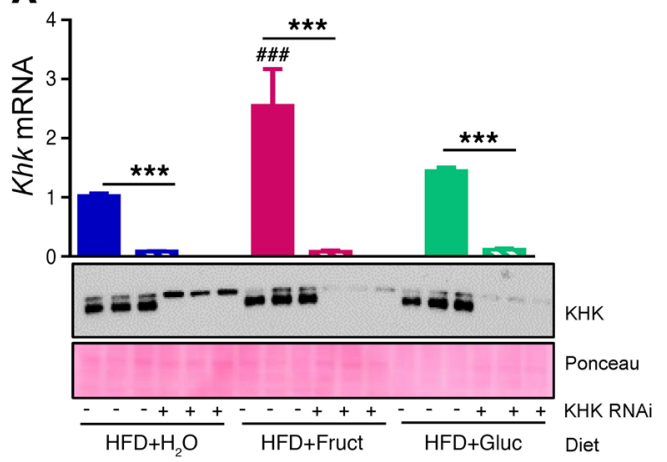

D

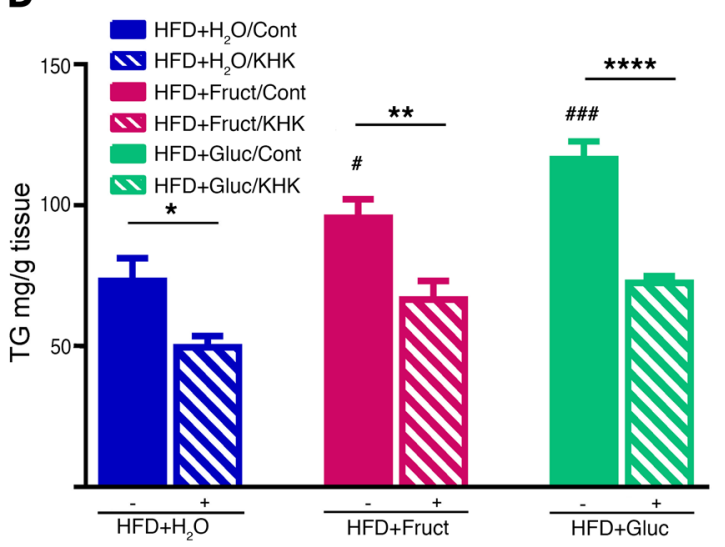

$\mathbf{F}$


B



E
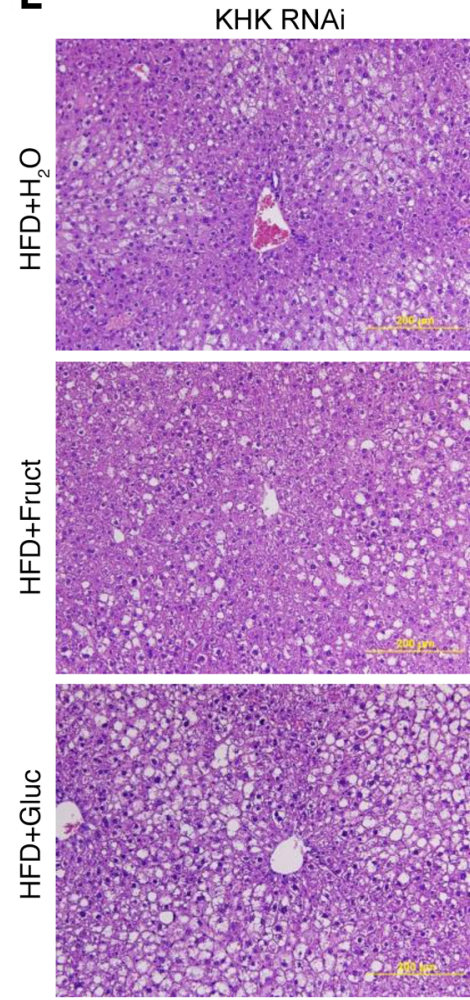

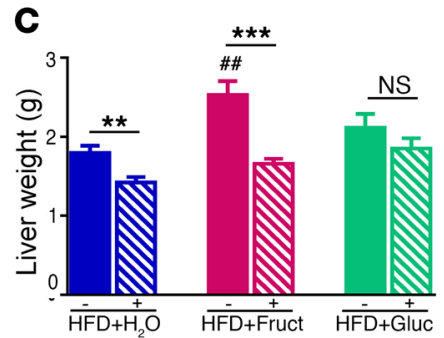

Scr RNAi
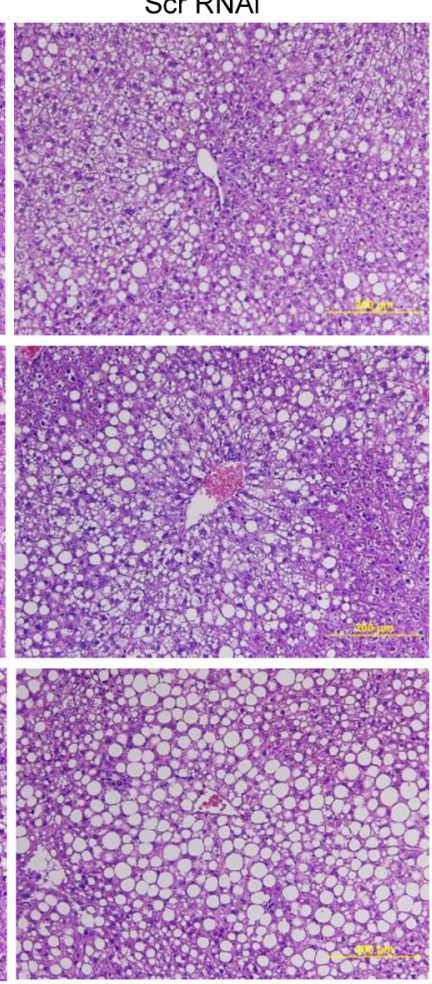

G

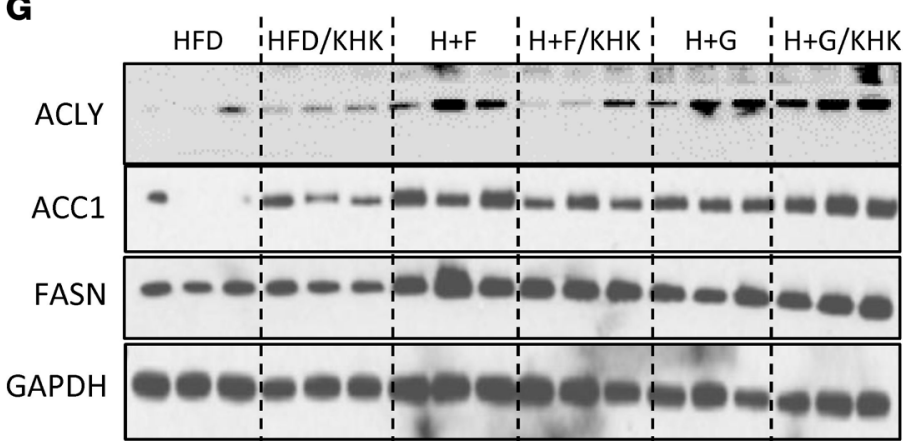

Figure 6. Khk knockdown improves liver steatosis. (A) Khk mRNA expression and protein levels in mice after 10 weeks on diets. Mice were treated with control or siRNA targeting KHK for the last 4 weeks. (B) Weight gain after 4 weeks of siRNA treatment and (C) liver weight after 6 weeks on diet followed by 4 weeks of siRNA treatment, while continuing on the same diets. (D) Liver triglyceride quantification and (E) histology in the same mice at sacrifice. Scale bars: $200 \mu \mathrm{m}$. (F) mRNA and (G) protein levels of enzymes regulating fatty acid synthesis after treatment with control or KHK targeting siRNA. Cont, control. $n=6$ mice per group. Statistical analysis was performed using 2-way ANOVA with post hoc $t$ tests between the individual groups. ${ }^{\#} P<0.05$; $\# P<0.01$;

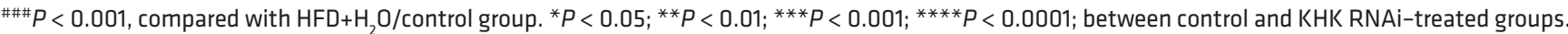

as both fructose and glucose increase Chrebp- $\beta$, which has been viewed as a marker of ChREBP activity (14), and knockout studies show that elimination of either SREBP1c (31) or ChREBP (32) contributes only to partial decrease in lipogenesis. While fructose and glucose upregulate different subsets of lipid synthetic and storage enzymes, HFD alone does not upregulate either of the lipogenic transcription factors, and in fact, the effects of fructose and glucose are attenuated on an HFD as compared with a chow diet. 
A



B



C

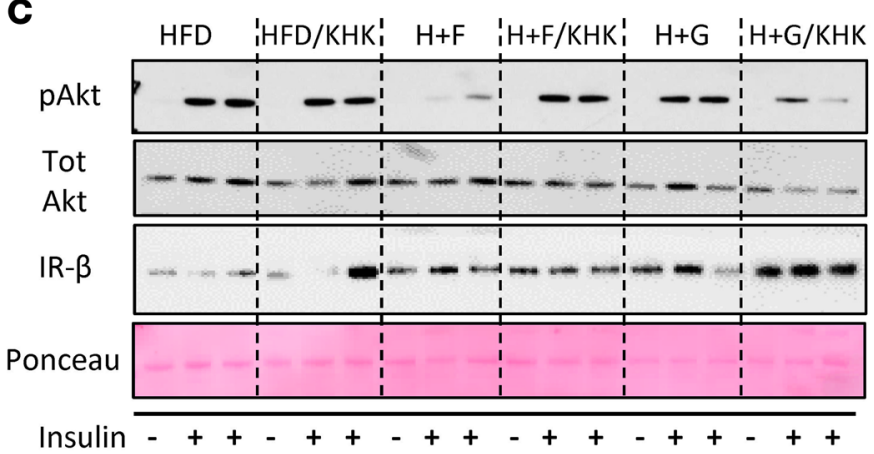

D

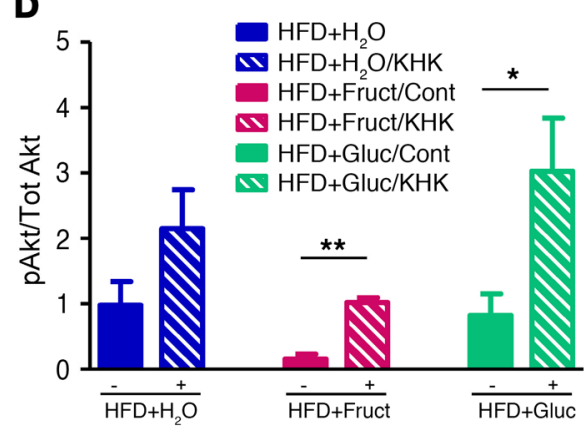

Figure 7. Khk knockdown improves glucose tolerance. Glucose tolerance in (A) HFD+Fruct and (B) HFD+H $\mathrm{O}$ and HFD+Cluc mice after 6 weeks on diets followed by 4 weeks of treatment with siRNA. (C) Western blot analysis and (D) ImageJ quantification of insulin signaling from livers of these mice. $n=6$ mice per group. Statistical analysis was performed using 2-way ANOVA with post hoc $t$ tests between the individual groups. ${ }^{*} P<0.05$; ${ }^{*} P<0.01$, between control and KHK RNAi-treated groups.

While both fructose- and glucose-supplemented mice on HFD developed hepatic steatosis, fructose supplementation resulted in increased intermediates of fatty acid synthesis in the liver, including increased acyl-CoA levels, whereas glucose-supplemented mice on HFD tended to have a higher expression of genes regulating liver triglyceride synthesis. In agreement with our results, a protective role of hepatic triglyceride accumulation has been proposed based on studies showing that overexpression of Dgat2, an enzyme that mediates the final step in TG synthesis, is associated with increased hepatic triglyceride accumulation, but not with insulin resistance (33). Conversely, knockdown of Dgat2 in genetically obese mice leads to a reduction of hepatic TG content, but a worsening of liver inflammation and fibrosis (34). Indeed human studies have also suggested that liver lipid composition, rather than the overall amount of lipids, is linked with progressive liver disease and obesity-associated metabolic abnormalities (35). In agreement with upregulation of different components of lipid synthesis and storage pathways, livers of fructose-supplemented mice show a mixture of microvesicular and macrovesicular steatosis, whereas glucose-supplemented mice show exclusive macrovesicular steatosis. Microvesicular steatosis has been linked with hepatocyte ballooning, mitochondrial dysfunction, and more severe NAFLD phenotype (36). Taken together, our data suggest that the difference in fructose- and glucose-induced lipogenesis stems from unique upregulation of a subset of lipogenic pathways, rather than a simple increase in the total amount of lipids.

Different monosaccharide supplementation also produced different levels of insulin resistance. Indeed, mice on HFD supplemented with glucose have improved fasting blood glucose and insulin levels as compared with mice on HFD alone or HFD plus fructose. The glucose-supplemented mice also had improved HOMA-IR and better glucose and insulin tolerance tests. This is most likely due to the fact that glucose supplementation on HFD is associated with salvage of insulin-stimulated Akt and IR- $\beta$ phosphorylation in the liver, as compared with fructose supplementation, which increased basal Akt and IR- $\beta$ phosphorylation. These changes in insulin signaling were also complemented by increases in expression of proteins associated with insulin resistance, such as $p 85 \alpha$ and Jnk with HFD and fructose supplementation, while glucose increased the expression of insulin receptor and Akt1, potentially improving insulin signaling. Additionally, glucosesupplemented mice on HFD had lower liver levels of branched chain amino acids, which could contribute to better insulin tolerance in these mice (16). Whereas an association between high fructose intake and worsening of insulin resistance has been previously reported (37), the effects of glucose supplementation on improving insulin resistance in mice on HFD are surprising. Our data also uniquely strengthen the association of fructose metabolism with insulin resistance by showing that siRNA-mediated knockdown of $K h k$, which reduces fructose metabolism specifically in the liver, can result in improved glucose tolerance and Akt phosphorylation in mice on HFD plus fructose diet and on HFD alone.

Previous reports suggest that consumption of foods with a high glycemic index is a risk factor for development of obesity 
and insulin resistance $(38,39)$, and pure glucose, by definition, has the highest glycemic index. However, glucose itself is not very sweet (40), and consumption of glucose-supplemented drinks is not equivalent to consumption of foods with a high glycemic index. In agreement with our findings in the mouse, Stanhope et al. found that when obese humans were given an ad libitum diet for 10 weeks and supplemented with glucose- versus fructosesweetened drinks, where the drinks provided $25 \%$ of energy requirements, those receiving fructose-sweetened drinks exhibited increased visceral adipose tissue and increased hepatic fatty acid synthesis, whereas the glucose-supplemented group had lower blood glucose levels and improved insulin sensitivity, albeit with increased serum triglycerides (41).

While fructose intake has been associated with the development of many metabolic complications, some challenge this hypothesis (42-44), as in many studies, fructose and glucose are often coingested in the form of sucrose or high-fructose corn syrup, precluding conclusions that one monosaccharide is more harmful than the other. Even studies exclusively utilizing fructosesweetened drinks to show this association have been contested, as fructose-sweetened drinks provide additional calories, which alone could explain the higher rates of obesity and metabolic complications. In fact, some studies suggest that fructose-increased caloric intake, mediated via leptin resistance (45) and antagonism of GLP-1R action in the brain (46), leads to increased adipogenic potential (47) and visceral adipose tissue inflammation (48). Our model controlled for additional calories ingested from sugarsweetened drinks by comparing the effects of fructose to those of isocaloric glucose, and while both monosaccharides resulted in higher total caloric intake, there was no difference in energy intake between the 2 sugar-supplemented groups. Thus, even in the setting of equivalent caloric intake, fructose-supplemented mice on HFD developed higher weight gain, increased percentage of visceral fat, augmented hepatic fatty acid synthesis, and hepatomegaly, resulting in insulin resistance, whereas the addition of glucose to the HFD was associated with increased gene expression of fatty acid oxidation and mitochondrial function genes, resulting in improved insulin sensitivity. Thus, our study demonstrates that, even with isocaloric intake, fructose ingestion is associated with poor metabolic outcomes, whereas glucose ingestion is not and can actually improve metabolism in the setting of HFD. Other studies utilizing HFD and high-fructose corn syrup supplementation (55\% fructose and $45 \%$ glucose) showed similar metabolic derangements (49), but our study suggests that it is fructose, but not glucose, metabolism that mediates these effects.

Fructose ingestion is associated with increased mRNA and protein expression of $\mathrm{KHK}$, the first enzyme of liver fructose metabolism. Interestingly, obese adolescents with NASH also have increased levels of KHK expression in the liver, a finding that needs to be confirmed in larger studies. These liver biopsies also showed elevated expression of enzymes regulating fatty acid synthesis, linking fructose intake with increased fatty acid synthesis. Increased fructose consumption $(50,51)$, augmented fatty acid synthesis (52), and elevated liver KHK levels (53) have been reported in adults with biopsy-proven NAFLD. Many studies also show that sugar consumption in children increases the risk of developing NAFLD (54), while fructose restriction decreases it (55-57); howev- er, KHK levels have not been previously examined in children with NAFLD or NASH. We report elevated KHK mRNA and protein levels in pediatric patients with NASH and show that knockdown of $K h k$ in the livers of mice is sufficient to improve hepatic steatosis, fatty acid synthesis, and whole body insulin resistance. In agreement with our results, Ishimoto et al. showed that whole body double-knockout of $K h k-A$ and $-C$ isoforms protects mice from steatohepatitis induced by HFD containing $30 \%$ calories from fructose (58). Interestingly, selective knockout of the $K h k-A$ isoform leads to increased hepatic lipid accumulation and worsening of glucose tolerance, presumably due to a secondary increase in fructose metabolism via the $K h k-C$ isoform (59). The $2 \mathrm{Khk}$ isoforms have different properties. Khk-C is mainly expressed in the liver, kidney, and intestine and has a high affinity for fructose, resulting in rapid metabolism, while $K h k-A$ is widely expressed and has a low affinity for fructose. We report, for what we believe is the first time, that the selective knockdown of $K h k$ in the liver is sufficient to ameliorate fructose-induced metabolic derangements. In contrast, knockout of glucokinase (Gck), the first enzyme of glycolysis in the liver, exacerbates hyperglycemia in mice (60). Thus, targeting fructose metabolism specifically in the liver utilizing siRNA may be a new strategy for treatment of patients with NAFLD, as siRNA gene-silencing technology is already used in other clinical studies $(61,62)$.

In summary, we show that consumption of fructose and glucose have very different health outcomes when superimposed on HFD. High fructose intake is associated with increased hepatic fatty acid synthesis and marked insulin resistance, while glucose supplementation leads to improved insulin signaling, despite producing a similar degree of hepatic steatosis. $K h k$ is increased in mice supplemented with fructose and in obese adolescent patients with NASH, whereas knockdown of Khk expression in mice leads to improvement in NAFLD and glucose tolerance. These results also suggest that substitution of dietary fructose with glucose may have beneficial metabolic effects, even in a setting of isocaloric intake.

\section{Methods}

Animals and diets. Mice were housed at $20-22^{\circ} \mathrm{C}$ on a 12 -hour light/ 12-hour dark cycle with ad libitum access to food and water. C57BL/6 male mice at 6 weeks of age were purchased from The Jackson Laboratory and fed either chow diet (Mouse Diet 9F, PharmaServ) or HFD (Research diets, D12492) for 10 weeks. Caloric composition of chow diet consisted of $23 \%$ protein, $21.6 \%$ fat, and $55.4 \%$ carbohydrates, while HFD had $20 \%$ protein, $60 \%$ fat, and $20 \%$ carbohydrates. Mice were watered with either tap water or $30 \%$ (wt/v) fructose or $30 \%$ glucose solution in water. Mice were weighed and their food intake was recorded once per week. In vivo metabolic parameters were measured utilizing CLAMS performed by Joslin Diabetes Center animal physiology core. Mice were sacrificed from 8 to $11 \mathrm{am}$, and 1 mouse from each cage, i.e., the dietary group, was utilized before sacrificing the next mouse in the same cage. This was repeated until all 4 mice per cage were sacrificed.

Glucose and insulin tolerance test. Oral glucose tolerance tests were performed after 8 weeks on diet. Mice were fasted overnight and gavaged with $2 \mathrm{mg} / \mathrm{g}$ of dextrose. Insulin tolerance tests were performed in nonfasted mice by i.p. injection of $1 \mathrm{mU} / \mathrm{kg}$ of insulin. Blood glucose levels were measured at $0,15,30,60$, and 120 minutes using a glucose meter (Infinity, US Diagnostics). 
Histology and triglyceride quantification. Tissues were stored frozen or fixed in formalin, and sections were stained with $\mathrm{H} \& \mathrm{E}$. Liver histology was graded by a board-certified veterinary pathologist blinded to the experimental conditions, and NAS was assessed as previously published (27). Triglycerides from liver samples were measured according to previously published methods (63). In brief, $100 \mathrm{mg}$ of liver tissue was homogenized in $1 \mathrm{ml}$ of chloroformmethanol $(2: 1 \mathrm{v} / \mathrm{v})$. Homogenate $(10 \mu \mathrm{l})$ was evaporated in glass tubes for 1 to 4 hours or overnight. Evaporated lipid was resuspended in $200 \mu \mathrm{l}$ of Triglycerides Reagent (Pointe Scientific, catalog T7532) and incubated for 15 minutes at room temperature; absorbance was measured at 500-nm wavelength. Concentration was extrapolated using $200 \mathrm{mg} / \mathrm{dl}$ triglyceride standard.

$m R N A, q P C R$, and RNA-seq analysis. mRNA was extracted by homogenizing liver tissue in TRIzol, treating with chloroform, and precipitating in 70\% ethanol. mRNA was purified using RNeasy Mini Kit columns (QIAGEN, catalog 74106). cDNA was made using High Capacity cDNA Reverse Transcription Kit (Applied Biosystems, cata$\log$ 4368813). qPCR was performed utilizing C1000 Thermal Cycler (BioRad, catalog CFX384). Primer sequences used are listed in Supplemental Table 1. HTG EdgeSeq mRNA sequence analysis was performed by the BioPolymers Facility at Harvard Medical School. Data were analyzed by the Bioinformatics Core at Joslin Diabetes Center. All original data were deposited in the NCBI's BioProject database (PRJNA391187).

Protein extraction, immunoblot, and immunoprecipitation. Tissues were homogenized in RIPA buffer (EMD Millipore) with protease and phosphatase inhibitor cocktail (BioTools). Cell fractionation was performed, when indicated, using NE-PER Nuclear and Cytoplasmic Extraction Kit (Thermo Scientific, catalog 78833). For immunoprecipitation, tissue was homogenized in nondenaturing buffer, $2 \mu \mathrm{g}$ of primary antibody was agitated overnight in a cold room, and protein was pulled with protein A/G magnetic beads (BioTools, catalog B23201). Proteins were separated using SDS-PAGE and transferred to PVDF membrane (Millipore). Immunoblotting was achieved using the indicated antibodies: acetylated-K (catalog 9441), p-Akt (catalog 4060), p-Erk1/2 (catalog 9101), p-IR (catalog L55B10), Tot Akt (catalog 11E7), lamin A/C (catalog 2032), ACLY (catalog 4332), ACACA (catalog 3662), FASN (catalog 3180), and SCD1 (catalog 2438) from Cell Signaling Technologies; IR (sc-711), p-Tyr (sc-7020), GAPDH (sc-25778), $\beta$-actin (sc-1616), and SREBP1 (sc-8984) from Santa Cruz Biotechnology Inc.; ChREBP (NB 400-135) from Novus; and KHK (catalog HPA007040) from Sigma-Aldrich. Quantification of immunoblots was performed using Image $(\mathrm{NIH})$.

Liver-specific Khk knockdown. Liver-specific knockdown was achieved utilizing an siRNA conjugated to $\mathrm{N}$-acetylgalactoseamine (GalNAc). Alnylam Pharmaceuticals synthesized siRNA to specifically target mouse Khk mRNA. The siRNA consists of 2 strands, guide and passenger. The guide strand carries the sequence information necessary for target-gene recognition, while the passenger strand supports loading into the RNA-induced silencing complex (RISC). siRNA has undergone chemical modifications to achieve long-lasting effect and specificity for hepatocytes. The combination of the 2'-fluoro, 2'-O-methyl and phosphorothioate modifications provides protection against exonuclease degradation, allowing for marked compound stabilization. The guide strand is conjugated to a trivalent GalNAc specifically recognized by the asialoglycoprotein receptor (ASGPR), which is highly expressed on the surface of hepatocytes, achieving hepatocyte-specific delivery and uptake. A GalNAc conjugate siRNA targeting transthyretin (TTR) was used as a negative control. Mice were injected subcutaneously with $20 \mathrm{mg} / \mathrm{kg}$ of KHK or control siRNA at 6 and 8 weeks on the diet and were sacrificed after 10 weeks on the diets.

Metabolomics analyses. Previously frozen liver tissue (50 mg) was homogenized in $1 \mathrm{ml}$ of $50 \%$ aqueous acetonitrile containing $0.3 \%$ formic acid. Liver amino acids, acyl CoAs, and organic acids were analyzed using stable isotope dilution techniques. Amino acid measurements were made by flow injection MS/MS using sample preparation methods described previously (15). The data were acquired using a Micromass Quattro Micro system equipped with a model 2777 autosampler, a model 1525 HPLC solvent delivery system, and a data system controlled by the MassLynx 4.1 operating system (Waters). Organic acids were quantified using methods described previously with Trace Ultra GC coupled to a Trace DSQ MS operating under Xcalibur 1.4 (Thermo Fisher Scientific) (64). Acyl CoAs were measured by LC-MS/MS (where LC indicates liquid chromatography) as described previously (65).

Statistics. All data are presented as mean \pm SEM. For experiments with only 2 groups, comparisons were made using $t$ tests. When a single variable was compared across more than 2 groups, for example, the data from human subjects, 1-way ANOVA was used. When experimental design included more than 2 groups, such as the effect of diet and sugar supplementation, the data were first analyzed using 2-way ANOVA and, for statistically significant overall results, post hoc $t$ tests were carried out to determine significant differences between the individual groups. Significant differences between diet types as compared with the Chow $+\mathrm{H}_{2} \mathrm{O}$ group were noted with a pound sign, and significant differences within the diet groups were designated by asterisks. For Figure 5E, due to lack of normality in human data, statistical analysis was performed on $\log _{2}$ transformed values.

Study approval. All animal studies were approved by the IACUC of the Joslin Diabetes Center and were in accordance with NIH guidelines. Approval was not needed for studies utilizing human liver biopsies, as the samples were received from the tissue repository in nonidentifiable form.

\section{Author contributions}

SS designed the experiments, performed the experiments, and wrote the manuscript. MKG, GXW, SF, BTON, and TNR helped perform the experiments and edited the manuscript. JW and KF provided siRNA, helped design the experiments, and edited the manuscript. $\mathrm{CH}$ graded liver histology and edited the manuscript. CBN and DEC helped design experiments, provided mentorship, and edited the manuscript. OI and CBN performed liver metabolomics profiling. CRK designed the experiments, provided mentorship, and wrote the manuscript.

\section{Acknowledgments}

The authors would like to thank Jonathan Dreyfuss and Hui Pan at the Joslin Diabetes Center Bioinformatics Core for analyzing RNA-seq data. We would also like to thank David Pober at the Joslin Diabetes Center and the Harvard Catalyst Biostatistics group for his help with statistical analysis. This work was supported in part by NIH grants R01 DK031036 and R01 DK033201 (to CRK); R01 DK056626, R01 DK103046, and R01 DK048873 (to DEC); K12 HD000850 (to SS); K08DK100543 (to BTON) and Joslin DRC grant P3ODK034834. This work was also supported, in part, by a grant from Alnylam Pharmaceuticals, awarded to SS and CRK. The human biopsy specimens 
used in this research were provided by the Pediatric Obesity Tissue Repository in the Center for Bariatric Research and Innovation at Cincinnati Children's Hospital Medical Center.
Address correspondence to: C. Ronald Kahn, Joslin Diabetes Center, One Joslin Place, Boston, Massachusetts 02215, USA. Phone: 617.732.2635; Email: c.ronald.kahn@joslin.harvard.edu.
1. Ford ES, Giles WH, Mokdad AH. Increasing prevalence of the metabolic syndrome among u.s. Adults. Diabetes Care. 2004;27(10):2444-2449.

2. Gotto AM, et al. The metabolic syndrome: a call to action. Coron Artery Dis. 2006;17(1):77-80.

3. Loomba R, Sanyal AJ. The global NAFLD epidemic. Nat Rev Gastroenterol Hepatol. 2013;10(11):686-690.

4. Ebbeling CB, et al. A randomized trial of sugar-sweetened beverages and adolescent body weight. N Engl J Med. 2012;367(15):1407-1416.

5. Ma J, et al. Sugar-sweetened beverage, diet soda, and fatty liver disease in the Framingham Heart Study cohorts. J Hepatol. 2015;63(2):462-469.

6. de Ruyter JC, Olthof MR, Seidell JC, Katan MB. A trial of sugar-free or sugar-sweetened beverages and body weight in children. $N$ Engl JMed. 2012;367(15):1397-1406.

7. Gaby AR. Adverse effects of dietary fructose. Altern Med Rev. 2005;10(4):294-306.

8. Nakagawa T, et al. A causal role for uric acid in fructose-induced metabolic syndrome. Am J Physiol Renal Physiol. 2006;290(3):F625-F631.

9. Softic S, Cohen DE, Kahn CR. Role of Dietary Fructose and Hepatic De Novo Lipogenesis in Fatty Liver Disease. Dig Dis Sci. 2016;61(5):1282-1293.

10. Chong MF, Fielding BA, Frayn KN. Mechanisms for the acute effect of fructose on postprandial lipemia. Am J Clin Nutr. 2007;85(6):1511-1520.

11. Lee AH, Scapa EF, Cohen DE, Glimcher LH. Regulation of hepatic lipogenesis by the transcription factor XBP1. Science. 2008;320(5882):1492-1496.

12. Horton JD, Goldstein JL, Brown MS. SREBPs: activators of the complete program of cholesterol and fatty acid synthesis in the liver. JClin Invest. 2002;109(9):1125-1131.

13. Postic C, Dentin R, Denechaud PD, Girard J. ChREBP, a transcriptional regulator of glucose and lipid metabolism. Annu Rev Nutr. 2007;27:179-192.

14. Kim MS, et al. ChREBP regulates fructoseinduced glucose production independently of insulin signaling. J Clin Invest. 2016;126(11):4372-4386.

15. An J, et al. Hepatic expression of malonyl-CoA decarboxylase reverses muscle, liver and whole-animal insulin resistance. Nat Med. 2004;10(3):268-274.

16. Newgard CB. Interplay between lipids and branched-chain amino acids in development of insulin resistance. Cell Metab. 2012;15(5):606-614.

17. Horton JD, Bashmakov Y, Shimomura I, Shimano $\mathrm{H}$. Regulation of sterol regulatory element binding proteins in livers of fasted and refed mice. Proc Natl Acad Sci USA. 1998;95(11):5987-5992.

18. Herman MA, et al. A novel ChREBP isoform in adipose tissue regulates systemic glucose metabolism. Nature. 2012;484(7394):333-338.

19. Bricambert J, Miranda J, Benhamed F, Girard J, Postic C, Dentin R. Salt-inducible kinase 2 links transcriptional coactivator $\mathrm{p} 300$ phosphorylation to the prevention of ChREBP-dependent hepatic steatosis in mice. JClin Invest. 2010;120(12):4316-4331.

20. Xu X, So JS, Park JG, Lee AH. Transcriptional control of hepatic lipid metabolism by SREBP and ChREBP. Semin Liver Dis. 2013;33(4):301-311.

21. Fisher FM, et al. A critical role for ChREBPmediated FGF21 secretion in hepatic fructose metabolism. Mol Metab. 2017;6(1):14-21.

22. Sae-Lee C, Moolsuwan K, Chan L, Poungvarin N. ChREBP regulates itself and metabolic genes implicated in lipid accumulation in $\beta$-cell line. PLOS ONE. 2016;11(1):e0147411.

23. Barbour LA, et al. Increased P85alpha is a potent negative regulator of skeletal muscle insulin signaling and induces in vivo insulin resistance associated with growth hormone excess. J Biol Chem. 2005;280(45):37489-37494.

24. Hirosumi J, et al. A central role for JNK in obesity and insulin resistance. Nature. 2002;420(6913):333-336.

25. Dominici FP, Hauck S, Argentino DP, Bartke A, Turyn D. Increased insulin sensitivity and upregulation of insulin receptor, insulin receptor substrate (IRS)-1 and IRS-2 in liver of Ames dwarf mice. J Endocrinol. 2002;173(1):81-94.

26. Asipu A, Hayward BE, O'Reilly J, Bonthron DT. Properties of normal and mutant recombinant human ketohexokinases and implications for the pathogenesis of essential fructosuria. Diabetes. 2003;52(9):2426-2432.

27. Kleiner DE, et al. Design and validation of a histological scoring system for nonalcoholic fatty liver disease. Hepatology. 2005;41(6):1313-1321.

28. de Koning L, Malik VS, Kellogg MD, Rimm EB, Willett WC, Hu FB. Sweetened beverage consumption, incident coronary heart disease, and biomarkers of risk in men. Circulation. 2012;125(14):1735-1741.

29. Pettinelli P, Videla LA. Up-regulation of PPAR-gamma mRNA expression in the liver of obese patients: an additional reinforcing lipogenic mechanism to SREBP-1c induction. J Clin Endocrinol Metab. 2011;96(5):1424-1430.

30. Benhamed F, et al. The lipogenic transcription factor ChREBP dissociates hepatic steatosis from insulin resistance in mice and humans. JClin Invest. 2012;122(6):2176-2194.

31. Liang G, Yang J, Horton JD, Hammer RE, Goldstein JL, Brown MS. Diminished hepatic response to fasting/refeeding and liver $\mathrm{X}$ receptor agonists in mice with selective deficiency of sterol regulatory element-binding protein-1c. J Biol Chem. 2002;277(11):9520-9528.

32. Dentin R, et al. Liver-specific inhibition of ChREBP improves hepatic steatosis and insulin resistance in ob/ob mice. Diabetes. 2006;55(8):2159-2170.

33. Monetti M, et al. Dissociation of hepatic steatosis and insulin resistance in mice overexpressing DGAT in the liver. Cell Metab. 2007;6(1):69-78.

34. Yamaguchi $\mathrm{K}$, et al. Inhibiting triglyceride synthesis improves hepatic steatosis but exac- erbates liver damage and fibrosis in obese mice with nonalcoholic steatohepatitis. Hepatology. 2007;45(6):1366-1374.

35. Puri P, et al. A lipidomic analysis of nonalcoholic fatty liver disease. Hepatology. 2007;46(4):1081-1090.

36. Tandra S, et al. Presence and significance of microvesicular steatosis in nonalcoholic fatty liver disease. J Hepatol. 2011;55(3):654-659.

37. Aeberli I, et al. Moderate amounts of fructose consumption impair insulin sensitivity in healthy young men: a randomized controlled trial. Diabetes Care. 2013;36(1):150-156.

38. Ludwig DS, Majzoub JA, Al-Zahrani A, Dallal GE, Blanco I, Roberts SB. High glycemic index foods, overeating, and obesity. Pediatrics. 1999;103(3):E26.

39. Ebbeling CB, Leidig MM, Sinclair KB, Hangen JP, Ludwig DS. A reduced-glycemic load diet in the treatment of adolescent obesity. Arch Pediatr Adolesc Med. 2003;157(8):773-779.

40. Steinert RE, Frey F, Töpfer A, Drewe J, Beglinger C. Effects of carbohydrate sugars and artificial sweeteners on appetite and the secretion of gastrointestinal satiety peptides. $\mathrm{Br} \mathrm{J} \mathrm{Nutr}$. 2011;105(9):1320-1328.

41. Stanhope KL, et al. Consuming fructosesweetened, not glucose-sweetened, beverages increases visceral adiposity and lipids and decreases insulin sensitivity in overweight/obese humans. J Clin Invest. 2009;119(5):1322-1334.

42. Tappy L, Lê KA. Metabolic effects of fructose and the worldwide increase in obesity. Physiol Rev. 2010;90(1):23-46.

43. White JS. Challenging the fructose hypothesis: new perspectives on fructose consumption and metabolism. Adv Nutr. 2013;4(2):246-256.

44. Rizkalla SW. Health implications of fructose consumption: A review of recent data. Nutr Metab (Lond). 2010;7:82.

45. Shapiro A, Mu W, Roncal C, Cheng KY, Johnson RJ, Scarpace PJ. Fructose-induced leptin resistance exacerbates weight gain in response to subsequent high-fat feeding. Am J Physiol Regul Integr Comp Physiol. 2008;295(5):R1370-R1375.

46. Burmeister MA, Ayala J, Drucker DJ, Ayala JE. Central glucagon-like peptide 1 receptor-induced anorexia requires glucose metabolismmediated suppression of AMPK and is impaired by central fructose. Am J Physiol Endocrinol Metab. 2013;304(7):E677-E685.

47. Zubiría MG, Alzamendi A, Moreno G, Rey MA, Spinedi E, Giovambattista A. Long-Term Fructose Intake Increases Adipogenic Potential: Evidence of Direct Effects of Fructose on Adipocyte Precursor Cells. Nutrients. 2016;8(4):198.

48. Marek G, et al. Adiponectin resistance and proinflammatory changes in the visceral adipose tissue induced by fructose consumption via ketohexokinase-dependent pathway. Diabetes. 2015;64(2):508-518.

49. Kohli R, et al. High-fructose, medium chain trans 
fat diet induces liver fibrosis and elevates plasma coenzyme $\mathrm{Q} 9$ in a novel murine model of obesity and nonalcoholic steatohepatitis. Hepatology. 2010;52(3):934-944.

50. Zelber-Sagi S, et al. Long term nutritional intake and the risk for non-alcoholic fatty liver disease (NAFLD): a population based study. J Hepatol. 2007;47(5):711-717.

51. Thuy $\mathrm{S}$, et al. Nonalcoholic fatty liver disease in humans is associated with increased plasma endotoxin and plasminogen activator inhibitor 1 concentrations and with fructose intake. J Nutr. 2008;138(8):1452-1455.

52. Lambert JE, Ramos-Roman MA, Browning JD, Parks EJ. Increased de novo lipogenesis is a distinct characteristic of individuals with nonalcoholic fatty liver disease. Gastroenterology. 2014;146(3):726-735.

53. Ouyang $\mathrm{X}$, et al. Fructose consumption as a risk factor for non-alcoholic fatty liver disease. JHepatol. 2008;48(6):993-999.

54. Papandreou D, Karabouta Z, Pantoleon A, Rousso I. Investigation of anthropometric, biochemical and dietary parameters of obese children with and without non-alcoholic fatty liver disease. Appetite. 2012;59(3):939-944.
55. Jin R, et al. Dietary fructose reduction improves markers of cardiovascular disease risk in HispanicAmerican adolescents with NAFLD. Nutrients. 2014;6(8):3187-3201.

56. Mager DR, Iñiguez IR, Gilmour S, Yap J. The effect of a low fructose and low glycemic index/load (FRAGILE) dietary intervention on indices of liver function, cardiometabolic risk factors, and body composition in children and adolescents with nonalcoholic fatty liver disease (NAFLD). JPEN J Parenter Enteral Nutr. 2015;39(1):73-84.

57. Lustig RH, et al. Isocaloric fructose restriction and metabolic improvement in children with obesity and metabolic syndrome. Obesity (Silver Spring). 2016;24(2):453-460.

58. Ishimoto T, et al. High-fat and high-sucrose (western) diet induces steatohepatitis that is dependent on fructokinase. Hepatology. 2013;58(5):1632-1643.

59. Ishimoto T, et al. Opposing effects of fructokinase $\mathrm{C}$ and $\mathrm{A}$ isoforms on fructose-induced metabolic syndrome in mice. Proc Natl Acad Sci U S A. 2012;109(11):4320-4325.

60. Postic C, et al. Dual roles for glucokinase in glucose homeostasis as determined by liver and pancreatic beta cell-specific gene knockouts using Cre recombinase. J Biol Chem. 1999;274(1):305-315.

61. Fitzgerald K, et al. Effect of an RNA interference drug on the synthesis of proprotein convertase subtilisin/kexin type 9 (PCSK9) and the concentration of serum LDL cholesterol in healthy volunteers: a randomised, single-blind, placebo-controlled, phase 1 trial. Lancet. 2014;383(9911):60-68.

62. Fitzgerald K, et al. A highly durable RNAi therapeutic inhibitor of PCSK9. N EnglJMed. 2017;376(1):41-51.

63. Softic S, et al. Lipodystrophy due to adipose tissue-specific insulin receptor knockout results in progressive NAFLD. Diabetes. 2016;65(8):2187-2200.

64. Jensen MV, et al. Compensatory responses to pyruvate carboxylase suppression in islet beta-cells. Preservation of glucose-stimulated insulin secretion. J Biol Chem. 2006;281(31):22342-22351.

65. White PJ, et al. Branched-chain amino acid restriction in Zucker-fatty rats improves muscle insulin sensitivity by enhancing efficiency of fatty acid oxidation and acyl-glycine export. $\mathrm{Mol}$ Metab. 2016;5(7):538-551. 